\title{
Ground-based measurements of the solar diameter during the rising phase of solar cycle 24
}

\author{
M. Meftah ${ }^{1}$, T. Corbard ${ }^{2}$, A. Irbah ${ }^{1}$, R. Ikhlef ${ }^{2,3}$, F. Morand ${ }^{2}$, C. Renaud ${ }^{2}$, A. Hauchecorne ${ }^{1}$, P. Assus ${ }^{2}$ J. Borgnino $^{2}$, \\ B. Chauvineau ${ }^{2}$, M. Crepel ${ }^{1}$, F. Dalaudier ${ }^{1}$, L. Damé ${ }^{1}$, D. Djafer ${ }^{4}$, M. Fodil ${ }^{3}$, P. Lesueur ${ }^{1}$, G. Poiet $^{1}$, M. Rouzé ${ }^{5}$, \\ A. Sarkissian ${ }^{1}$, A. Ziad ${ }^{2}$, and F. Laclare ${ }^{2}$
}

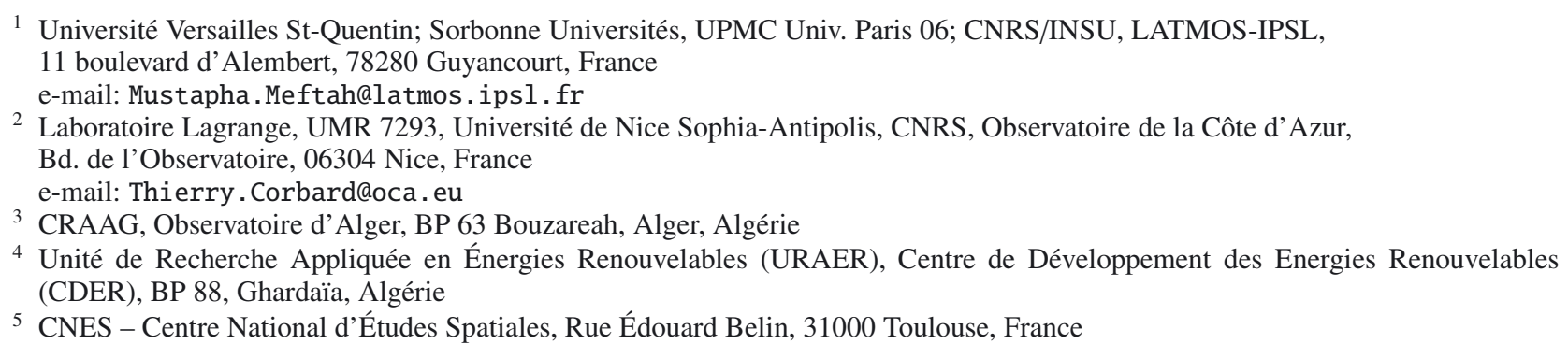

Received 7 February 2014 / Accepted 5 August 2014

\section{ABSTRACT}

\begin{abstract}
Context. For the past thirty years, modern ground-based time-series of the solar radius have shown different apparent variations according to different instruments. The origins of these variations may result from the observer, the instrument, the atmosphere, or the Sun. Solar radius measurements have been made for a very long time and in different ways. Yet we see inconsistencies in the measurements. Numerous studies of solar radius variation appear in the literature, but with conflicting results. These measurement differences are certainly related to instrumental effects or atmospheric effects. Use of different methods (determination of the solar radius), instruments, and effects of Earth's atmosphere could explain the lack of consistency on the past measurements. A survey of the solar radius has been initiated in 1975 by Francis Laclare, at the Calern site of the Observatoire de la Côte d'Azur (OCA). Several efforts are currently made from space missions to obtain accurate solar astrometric measurements, for example, to probe the long-term variations of solar radius, their link with solar irradiance variations, and their influence on the Earth climate.

Aims. The Picard program includes a ground-based observatory consisting of different instruments based at the Calern site (OCA, France). This set of instruments has been named "Picard Sol" and consists of a Ritchey-Chrétien telescope providing full-disk images of the Sun in five narrow-wavelength bandpasses (centered on 393.37, 535.7, 607.1, 782.2, and $1025.0 \mathrm{~nm}$ ), a Sun-photometer that measures the properties of atmospheric aerosol, a pyranometer for estimating a global sky-quality index, a wide-field camera that detects the location of clouds, and a generalized daytime seeing monitor allowing us to measure the spatio-temporal parameters of the local turbulence. Picard Sol is meant to perpetuate valuable historical series of the solar radius and to initiate new time-series, in particular during solar cycle 24.

Methods. We defined the solar radius by the inflection-point position of the solar-limb profiles taken at different angular positions of the image. Our results were corrected for the effects of refraction and turbulence by numerical methods.

Results. From a dataset of more than 20000 observations carried out between 2011 and 2013, we find a solar radius of $959.78 \pm 0.19 \operatorname{arcsec}(696113 \pm 138 \mathrm{~km})$ at $535.7 \mathrm{~nm}$ after making all necessary corrections. For the other wavelengths in the solar continuum, we derive very similar results. The solar radius observed with the Solar Diameter Imager and Surface Mapper II during the period 2011-2013 shows variations shorter than 50 milli-arcsec that are out of phase with solar activity.
\end{abstract}

Key words. astrometry - Sun: fundamental parameters - Sun: activity

\section{Introduction}

Measurements of the solar radius are of great interest within the scope of the debate on the role of the Sun in climate change (Rozelot 2001a,b; Schröder 2001). The solar radius is mainly related to the knowledge of the solar atmosphere. However, it is very difficult to measure this fundamental parameter of astrophysical interest. Solar radius determination is one of the oldest problems in astrophysics. Systematic measurements of the solar radius have been made since Antiquity (Rozelot \& Damiani 2012). At the end of the nineteenth century, an investigation of the value of the solar radius obtained by meridian observations was carried out by Arthur Auwers (Auwers 1891). He published a value for the solar radius of 959.63 arcsec that he derived from heliometer measurements made by 29 observers (Wittmann 1977) during the period 1873-1886. In solar modeling, this canonical value has been commonly used and was adopted by the International Astronomical Union (IAU). Solar radius measurements (mostly from ground) are plotted in Fig. 1, showing inconsistent results that are probably caused by different instruments, different spectral domains of measurements, different calculation methods, different definitions (Haberreiter et al. 2008b), and different sites where the conditions of observations are not comparable. Thus, the accurate absolute value of the solar radius is not the subject of a consensus. Indeed, the Earth 

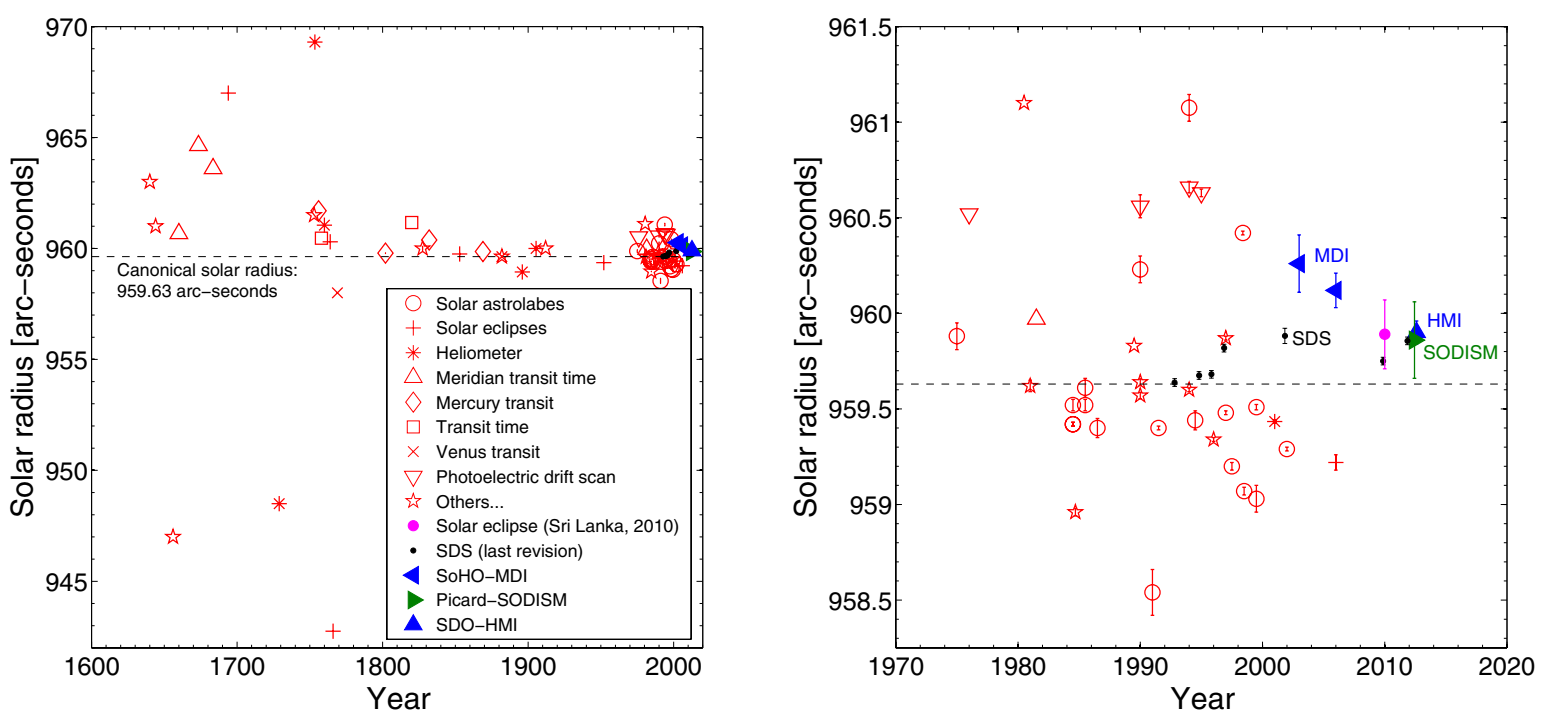

Fig. 1. Left: solar radius measurements (red symbols) made since the seventeenth century (Rozelot \& Damiani 2012). The mean value of all these measurements is close to 960 arcsec. Right: focus on solar radius measurements made since 1970. Solar Disk Sextant (SDS) measurements (Sofia et al. 2013) are represented with black circles. Solar and Heliospheric Observatory - Michelson Doppler Imager (SoHo-MDI) and Solar Dynamics Observatory - Helioseismic and Magnetic Imager (SDO-HMI) records are represented with blue symbols. The Solar Diameter Imager and Surface Mapper (SODISM) measurement obtained during the transit of Venus is represented with a green symbol. Adassuriya et al. (2011) found a solar radius of $959.89 \pm 0.18$ arcsec (see magenta circle symbol) during solar eclipse in Sri Lanka.

atmosphere generates various hindrances that make morphometric and photometric studies subject to discussion concerning the distinction between solar activity and atmospheric effects merged in ground-based measurements. They include refraction, turbulence, scattering, extinction, and diurnal alternation. It is suspected that the past inconsistencies of the temporal dependence of the solar radius measured from the ground stem primarily from such contingencies (Ribes et al. 1991; Delache \& Kroll 1994; Badache-Damiani et al. 2007). Efforts have been made in the past to understand and quantify the effects of atmospheric disturbances on ground-based observations (Brown 1982; Lakhal et al. 1999). The interpretation of ground-based observations, however, remains controversial to date, and recent measurements obtained outside the atmosphere (balloon flights and space instruments) indicate that the canonical value of the solar radius is under-estimated. Ideally, space instrumentation is required for solar radius measurements, but this instrumentation is a high-level technical challenge given the desired accuracy (a few milli-arcsec), and mission duration in a harsh environment (BenMoussa et al. 2013). From the ground, the instruments are not affected by degradation due to space environment, and maintenance can be easily provided. If, in addition, the atmospheric effects are properly monitored and taken into account, they represent our best chance to build the needed long time-series records. That is why an important program of measurements from the ground is associated with the space operations during and after the Picard mission. The Picard program (Thuillier et al. 2006) owes its name to Jean Picard, considered as a pioneer of precise modern astrometry. Picard Sol comprises the Solar Diameter Imager and Surface Mapper II or SODISM II (a copy of the SODISM space instrument), the turbulence monitor (Moniteur d'Images Solaires Franco-Algérien or MISOLFA), and the additional instrumentation. The main objectives of the Picard Sol mission are:

- to understand the influence of the atmosphere on the solar radius;
- to determine the relation between the turbulence parameters and the measured solar radius;

- to determine whether small-angle scattering by aerosols could also impact significantly the metrologic accuracy;

- to compare the solar radius measurements obtained with SODISM II and ground-based instruments (to identify possible biases);

- to continue solar radius measurements with ground-based instruments.

\section{Historical solar radius measurements at Calern Observatory}

The solar radius survey was initiated in 1975 with the Solar Astrolabe at Calern Observatory (France). Simultaneously with visual observations on the same instrument, a program of charge-coupled device (CCD) records was conducted, which started in 1989. The coherence of visual and CCD measurements thus obtained over ten years permitted qualifying the whole visual series, which appeared to be free of systematic personal effects (Laclare et al. 1999). The DORAYSOL (Définition et Observation du Rayon Solaire) instrument was then designed and also developed at Calern Observatory. The principle of this instrument remains the same as that of the Solar Astrolabe (timing the crossing of a parallel of altitude by the Sun), but a prism at a varying angle enables more daily measurements. The DORAYSOL mean solar radius of 959.48 arcsec (with $\sigma=0.32$ arcsec) was deduced from 19169 measurements between years 2000 and 2006 (Morand et al. 2010). Data were corrected for atmospheric refraction, but not for turbulence. For the same period, a mean solar radius of 959.55 arcsec (with $\sigma=0.26$ arcsec) was deduced from 371 Astrolabe measurements (with the same corrections). Measurements of the solar radius made at Calern with the Solar Astrolabe over two solar cycles (between 1978 and 1994) show apparent variations anticorrelated with solar activity defined by the number of sunspots (Laclare et al. 1996). These results have raised many questions 
and led to the development of the dedicated Picard mission. Simulation of atmospheric effects on the solar radius measurements made at the Solar Astrolabe also showed the influence of seeing conditions and the importance of having a monitor that records the image quality (Lakhal et al. 1999). The idea of developing MISOLFA (Irbah et al. 2001; Assus et al. 2002) established itself, to run in conjunction with the Picard space mission and SODISM II. Thus, the Picard program contributes to the historical solar radius series initiated at Calern Observatory.

\section{Solar radius variability}

Possible temporal variations of the solar radius are important as an indicator of internal energy storage and as a mechanism for changes in the total solar irradiance (TSI). Long-term or cyclic variations in the solar luminosity can be related to the corresponding changes in the solar radius $R_{\odot}$ and effective temperature $T_{\text {eff }}$ of the Sun by derivating the Stefan-Boltzmann equation:

$$
\frac{\Delta \mathrm{TSI}}{\mathrm{TSI}}=2 \times \frac{\Delta R_{\odot}}{R_{\odot}}+4 \times \frac{\Delta T_{\mathrm{eff}}}{T_{\mathrm{eff}}} .
$$

Thus, it is interesting to estimate the solar radius variations with the solar cycle. If we assume that the observed TSI variations over a solar cycle $(\sim 0.1 \%)$ represents an upper limit for the luminosity variation and assuming no variation in $T_{\mathrm{eff}}$, the strongest possible variation $\Delta R_{\odot}$ of the solar radius cannot exceed 0.5 arcsec during a solar cycle. Periodicities of solar activity with periods longer than the sunspot cycle (e.g., the 87 year Gleissberg cycle or the 210 year Suess cycle) have been found and fueled a discussion on the influence of solar variability on the Earth's climate (Braun et al. 2005). The potential link between solar activity and solar radius variations remains a matter of debate, however, requiring both modeling and measurements with enough accuracy over long periods. The relationship between the solar radius and the solar activity is the field of measurements and solar modeling. One of the first researchers to raise questions about the solar radius variations was Hermann Helmholtz (1821-1894), who proposed a theory for the solar luminosity that states that the Sun had been larger in the past and was slowly collapsing into itself because of its own gravity, releasing gravitational energy in the form of light and heat. But that was before nuclear fusion was discovered. Eddy \& Boornazian (1979) were pioneers in the field of measuring solar radius variations. From the analysis of Greenwich meridian transit measurements over more than a century (1836-1853), they found a statistically significant secular decrease of the solar diameter of about $0.1 \%$ per century, which is even more than the rate proposed by Helmholtz in 1854 to explain solar luminosity. Later, however, while analyzing larger datasets spanning 265 years, Gilliland (1981) reported a marginally significant secular decrease of only around 0.1 arcsec per century. Other analyses concluded that there has been no detectable variation of the Sun over the past 250 years (Parkinson et al. 1980). Michel Toulmonde compiled all the solar radius measurements made between 1660 to 1995 and concluded that the mean solar radius at one astronomical unit is $960.0 \pm 0.1$ arcsec. His investigations did not reveal any substantial secular variation in the solar radius (Toulmonde 1997). However, it is very difficult to extract a trend in the solar radius from historical data. Thus, a possible long-term trend in solar radius records is still a matter of debate. On the one hand, the variability of the solar radius can be analyzed during a solar cycle. Historical solar radius measurements performed at the Calern site have revealed an anticorrelation with solar activity (Laclare et al. 1996) for a period covering solar cycles 21 and 22 (19781994). However, during solar cycle 23, ground-based records (Solar Astrolabe and DORAYSOL) showed no clear correlation or anticorrelation between the solar radius and the activity of the Sun (Morand et al. 2010). The Solar Disk Sextant (SDS) experiment shows the solar radius variability through its seven balloon flights during the years 1992 to 2011. The solar radius is found to vary over that period by up to $0.2 \operatorname{arcsec}$ (Sofia et al. 2013), but the variation is not in phase with solar activity. Data from the Michelson Doppler Imager (MDI) instrument onboard the Solar and Heliospheric Observatory (SoHo) cover the whole solar cycle 23 and show no evidence of secular trends in the solar radius, or variations attributable to the 11 year cycle. Systematic changes in the solar radius with the sunspot cycle must be smaller than 23 milliarcsec (mas) peak-to-peak (Kuhn et al. 2004; Bush et al. 2010). Solar radius variations for different instruments are plotted in Fig. 2, showing some inconsistent results. The MDI result, even if often considered as the most reliable to date, would therefore certainly gain in being confirmed by other dedicated solar missions, such as Picard. Foukal et al. (2006) concluded that it is unlikely that solar radius measurements can reveal deeper-lying sources of solar irradiance variations, as was originally hoped. This is coherent with both the fact that modeling the net contribution of sunspots, faculae and plages is able to explain at least $90 \%$ of the observed cycle TSI variations (Ball et al. 2012), and the fact that no observable solar radius variation is expected from these surface effects (Spruit 1991). Therefore, changes in the size of the solar disk contribute probably negligibly to the TSI variations during a solar cycle. Coherent long-term measurements are therefore still needed to determine any significant variations of the solar radius during a solar cycle or any secular trend. This is the aim of the Picard SOL project in continuation of the series started at Calern observatory in 1978.

\section{Picard Sol, a ground-based facility for long-term measurements}

The Picard program includes a ground-based observatory consisting of different instruments based at the Calern site (Observatoire de la Côte d'Azur, France). Picard Sol and its instruments were described in detail by Meftah et al. (2012). SODISM II is a multiwavelength full disk solar imager specially designed for metrological solar radius measurements. MISOLFA (Corbard et al. 2010; Irbah et al. 2010; Ikhlef et al. 2012) is a high-cadence solar limb imager allowing us to measure the spatio-temporal parameters of the local turbulence. The photometer provides a quality index of pictures taken by SODISM II (aerosol optical depth or thickness and water vapor). The pyranometer measures the luminous flux received and provides another quality index for SODISM II measurements. The visible wide-field camera is used to detect the location of clouds.

\subsection{The SODISM II ground-based instrument and solar radius measurements}

For the space mission, two identical units were developed. One unit named SODISM (Meftah et al. 2014b) was launched on 15 June 2010, and the second was installed some months later at Calern Observatory (N 43 44'53" latitude, E $6^{\circ} 55^{\prime} 36^{\prime \prime}$ longitude and altitude of $1271 \mathrm{~m}$ ). This ground-based unit, named SODISM II, is placed in a vacuum tank (Fig. 3) and pointed toward the Sun. 


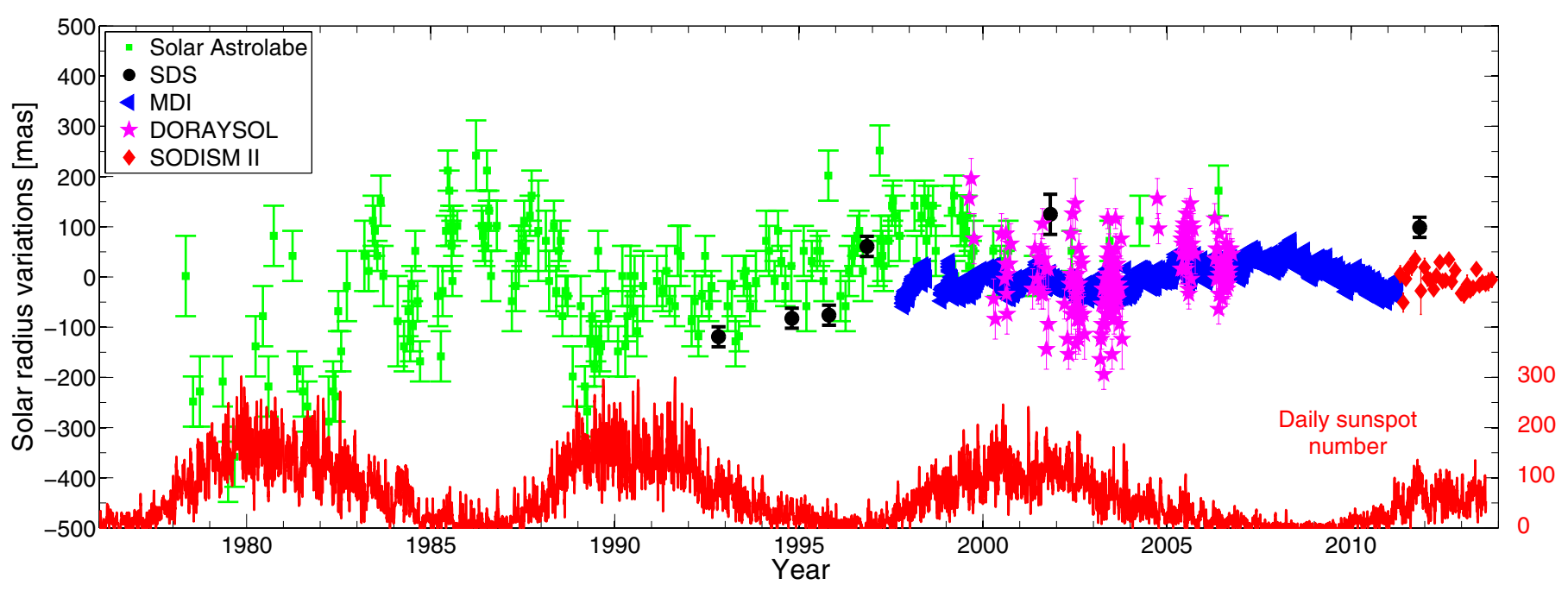

Fig. 2. Evolution of the solar radius variations over time for ground instruments (Solar Astrolabe, DORAYSOL and SODISM II monthly mean at $782.2 \mathrm{~nm}$ ), balloon experiment (SDS), and space instrument (MDI) vs. daily sunspot number time-series. For each series, the mean has been taken as reference value.

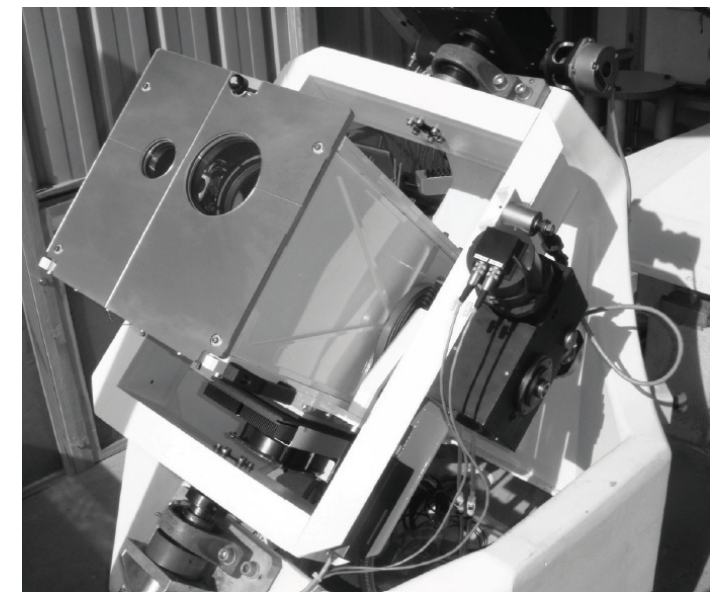

Fig. 3. View of the SODISMII equatorial mount. The ground-based telescope is placed in a vacuum tank closed by a glass window.

SODISM II is an $11 \mathrm{~cm}$ diameter telescope with a CCD at its focal plane. One image is recorded every minute with the instrument. It is a Ritchey-Chrétien telescope (to minimize both spherical and coma aberrations) with a focal length of $2626 \mathrm{~mm}$, and an aperture of $\mathrm{F} / 23$ with a central obscuration of $40 \%$ in diameter $(\mathrm{F} / 30$ and $50 \%$ for the main channel). This aperture was chosen for geometric resolution. The field of view is $\sim 36 \times 36$ arcmin. The detector is a 2048 by 2048 pixel CCD array with square $13.5 \mu \mathrm{m}$ pixels $(\sim 1.06$ arcsec per pixel). During nominal operations (solar pointing), the Sun is the only significant light source and it almost fills the field of view. This is why no external baffles were foreseen. This design has some field curvature, but image quality is only required at the Sun edge, so the focus is adjusted to this. The SODISM II main optical path consists essentially of a front window, a primary mirror (M1), a secondary mirror (M2), interchangeable interference filters, and a CCD. These choices about the optical configuration and spectral channels determine the core design of SODISM II, but the instrument has been supplemented with a number of important design features that augment its capacities:

1. the whole SODISM II instrument is in primary vacuum at $\sim 10$ mbar;
2. the whole SODISM II assembly is thermally regulated at $\sim 20^{\circ} \mathrm{C}$;

3. the Sun image is stabilized on the detector by the equatorial mount (better than \pm 0.5 arcsec during the exposure time);

4. a front window bears a reflective and absorbing coating on its inner side, which divides the penetration of solar flux inside the instrument by $\sim 20$;

5. two successive filter wheels permit inserting one of the spectral filters (between 393.37 and $1025.0 \mathrm{~nm}$ ) and other refractive elements (lens for stellar operations), or to leave the optical path open;

6. a $2 k \times 2 k$ frame-transfer CCD is placed at the focal plane. A shutter mechanism provides it with dark conditions except within the duration of its electronic exposures. The CCD is anti-reflective coated;

7. a Peltier and a cold finger remove the heat at the back of the CCD to cool it $\left(-10{ }^{\circ} \mathrm{C} \pm 0.2{ }^{\circ} \mathrm{C}\right)$ and decrease its darksignal during nominal operations. The low CCD temperature and the dark conditions granted by the shutter provide the relatively slow readout of the camera $(\sim 22 \mathrm{~s})$.

Two filters wheels carry a set of interference filters with specific roles, as shown in Table 1 . The central wavelengths of the five spectral bands (viz. 393.37, 535.7, 607.1, 782.2 nm, and $1025.0 \mathrm{~nm}$ ) ensue from the following rationale. The photospheric bandpasses ( 535.7 to $1025.0 \mathrm{~nm}$ ) were selected for being quasi-free of Fraunhofer lines to account for the solar continuum and to therefore neglect any overlying structure. They also had to perpetuate valuable historical series, or to initiate new time-series if a compelling case could be made. A narrow bandpass centered around $535.7 \mathrm{~nm}$ was chosen for its heritage with the Calern measurements (Solar Astrolabe and DORAYSOL), although they were carried out in a wider bandpass of around $548 \mathrm{~nm}$ (Laclare et al. 1996). SODISM II is a replica of the space instrument and used two different filters at $535.7 \mathrm{~nm}$ for solar astrometry (a) and helioseismic observations (b). Furthermore, SODISM probes the solar interior via an helioseismic analysis of the solar disk and limb images at $535.7 \mathrm{~nm}$ (b), and via astrometric investigations at the limb. For our ground-based measurements, we kept these two filters at $535.7 \mathrm{~nm}$ to monitor the aging process. In both cases, we are beyond the characteristic time of the turbulence, which is 
Table 1. SODISM II channels, characteristics, and relationship with other instruments.

\begin{tabular}{lcccl}
\hline \hline Wavelength & Solar atmosphere & Bandwidth [nm] & Exposure time [s] & Role/relationship \\
\hline $393.37 \mathrm{~nm}$ & Ca II K line & $\sim 0.7$ & 1.70 & Chromosphere influence \\
$535.7 \mathrm{~nm}$ (a) & Continuum & $\sim 0.5$ & 1.30 & Solar Astrolabe and DORAYSOL \\
$535.7 \mathrm{~nm}$ (b) & Continuum & $\sim 0.5$ & 8.90 & Solar Astrolabe and DORAYSOL \\
$607.1 \mathrm{~nm}$ & Continuum & $\sim 0.7$ & 1.28 & PSPT, SDS, SDO-HMI \\
$782.2 \mathrm{~nm}$ & Continuum & $\sim 1.6$ & 1.43 & SDM \\
$1025.0 \mathrm{~nm}$ & Continuum & $\sim 6.4$ & 1.70 & New time-series \\
\hline
\end{tabular}

Table 2. Solar radius observations in $\operatorname{arcsec}\left({ }^{\prime \prime}\right)$ at one astronomical unit (AU).

\begin{tabular}{|c|c|c|c|}
\hline Instrument, site, authors & Years & Solar radius $\left[{ }^{\prime \prime}\right]$ & $\lambda[\mathrm{nm}]$ \\
\hline Solar Astrolabe, Calern (Fr), Laclare et al. (1999) & $1975-1998$ & $959.51 \pm 0.01^{*}$ & 540.0 \\
\hline SDM, Boulder (US), Brown \& Christensen-Dalsgaard (1998) & 1981-1987 & $959.65 \pm 0.01^{* *}$ & 800.0 \\
\hline SDS, Ba & 1992-2011 & $959.76 \pm 0.01$ & 615.0 \\
\hline DO & 1999-2006 & 959. & 548.0 \\
\hline Sol & 2003,2006 & .09 & 676.78 \\
\hline 4a) & 2012 & 959. & 607.1 \\
\hline SDO-HMI, in space (Transit of Venus), Hauchecorne et al. (2014) & 2012 & $959.90 \pm 0.06$ & 617.3 \\
\hline
\end{tabular}

Notes. ${ }^{(*)}$ Solar Astrolabe and DORAYSOL data were corrected for atmospheric refraction and for zenith distance, but not for turbulence. ${ }^{(* *)}$ Corrected data to one astronomical unit.

measured in milliseconds. A second narrow channel was adopted at $607.1 \mathrm{~nm}$. It can be compared with the 590-670 nm spectral range of the SDS balloon experiment (Sofia et al. 1984, 2013). This band has been used by the Precision Solar Photometric Telescope (PSPT) and produces images at $607.1 \mathrm{~nm}$ (Coulter et al. 1996) in the red continuum ${ }^{1}$. It can also be compared with the $617.3 \mathrm{~nm}$ continuum spectral range of the SDO-HMI space experiment (Scherrer et al. 2012; Schou et al. 2012). A third Fraunhofer-line-free bandpass is centered on $782.2 \mathrm{~nm}$. It can relate to the Solar Diameter Monitor (SDM) measurements at $800 \mathrm{~nm}$ (Brown \& Christensen-Dalsgaard 1998). A last Fraunhofer-line-free bandpass is centered on $1025.0 \mathrm{~nm}$. With this wavelength, we initiate a new time-series, and while remaining in the photospheric continuum, we hope to minimize the impact of turbulence for ground-based observations. The chromospheric channel is centered on $393.37 \mathrm{~nm}$ (Ca II K line, singly ionized calcium, which is magnetically active). This permits us to image the low chromosphere and particularly, to observe the regions with an enhanced contrast. It can be compared with the PSPT experiment. References to solar radius observations corresponding to our channels are listed in the Table 2. It is important to note, however, that the uncertainties quoted by the authors for each set of measurements are not directly comparable because they cover periods of different lengths and do not all include estimates of the contribution from systematic effects.

SODISM II has recorded more than 75000 solar images since the beginning of the mission in May 2011. Figure 4 shows a sample of Level-1 solar images at the six wavelengths recorded in 2013. All images were corrected for dark-current and flat-field. Thus, we can track the solar radius.

\subsection{The MISOLFA instrument and turbulence monitoring}

Simulation of atmospheric effects on the solar radius measurements shows the influence of seeing conditions (Lakhal et al. 1999). There are three common descriptions of the astronomical seeing conditions at an observatory of (i) the Fried parameter $r_{0}$ (size of a typical lump of uniform air within the turbulent

\footnotetext{
1 http://lasp.colorado.edu/pspt_access/
}

atmosphere) and $\tau_{0}$ (the time-scale over which the changes in the turbulence become significant); (ii) the turbulence vertical profile $C_{n}^{2}(h)$; and (iii) the full width at half maximum (FWHM) of the seeing disk. Using the Von Kàrmàn's model, it is shown that turbulence simulated through the Fried parameter $\left(r_{0}\right)$ modifies the measured solar radius (Ikhlef et al. 2012). Figure 5 presents the effect of turbulence as a function of $r_{0}$ on SODISM II solar radius for each wavelength and shows that the systematic bias decreases as $r_{0}$ increases. At $535.7 \mathrm{~nm}$, we note that for $r_{0}$ equal to $7 \mathrm{~cm}$, the asymptotic value is reached, but with a bias of $\sim 0.18$ arcsec. Figure 6 shows the limb-shape evolution for different values of $r_{0}$ (FWHM of the solar limb first derivative decreases as $r_{0}$ increases). This indicator may also be used to correct the solar radius measurements obtained by SODISM II (see also Fig. 13). Thus, atmospheric turbulence modifies the ground-based observed shape of the solar limb, and this effect is always considered to be the source of the discrepancies among the radius determinations. These numerical results confirmed turbulence as a perturbing phenomenon that needs correcting for by about few tens of arcsec, and led to build a dedicated instrument (MISOLFA) to measure the turbulence parameters. MISOLFA is a solar seeing monitor associated with a CCD detector at its focal plane. This instrument is placed on an Alt-Azimuth mount support, which rotates MISOLFA about two perpendicular axes. The instrument has been described in detail by Irbah et al. (2010). Thirty-two solar limbs are recorded every second with the instrument. It is a Cassegrain-coudé telescope with a focal length of $10000 \mathrm{~mm}$, a main entrance pupil of $252 \mathrm{~mm}$, and an aperture of $\mathrm{F} / 40$. The field of view is $\sim 2.1 \times 1.6$ arcmin. The detector is a 640 by 480 pixel CCD array with square $9.9 \mu$ m pixels $(\sim 0.20 \operatorname{arcsec}$ per pixel). The MISOLFA design is based on the statistical analysis of the entry angle fluctuations defined as the slope in each point of the wavefront through the pupil. In the case of diurnal conditions, these fluctuations are shown by the observation of the solar limb. Two measurement channels are provided:

1. a direct channel in which the Sun image is formed on a CCD camera with suitable magnification. This channel enables the evaluation of the spatial coherence parameters of 


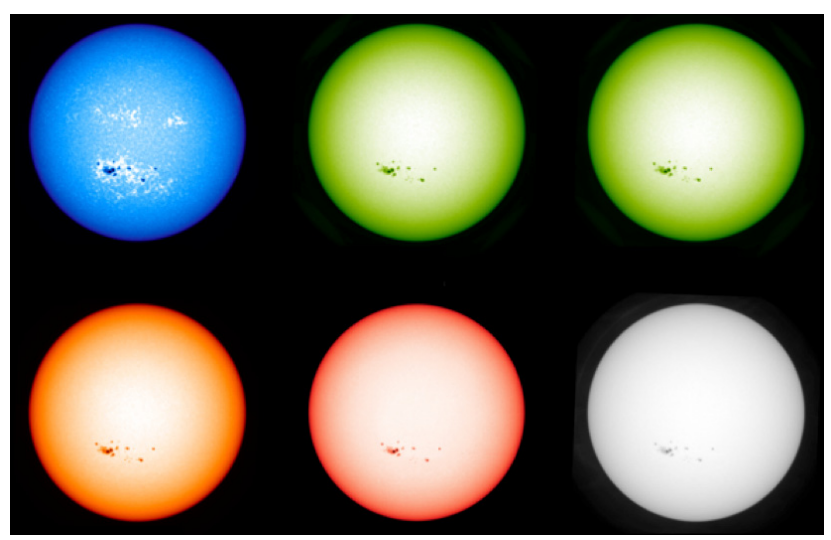

Fig. 4. Top: solar images in the different SODISM II channels (Level-1 data products): $393.37,535.7$ (a) and $535.7 \mathrm{~nm}$; (b) starting from left to right. Bottom: solar images for other wavelengths: 607.1, 782.2 and $1025.0 \mathrm{~nm}$ starting from left to right.

the wavefront (Fried parameter, outer scale and isoplanetism domain) as well as the turbulence profiles;

2. a second channel (pupil channel) that forms the image of the pupil through a diaphragm placed on the solar limb is used to evaluate the wavefront temporal parameters using photo-electric detectors (photodiodes).

The first MISOLFA objective is to quantify the atmosphere effects on the ground-based solar radius measurements made by SODISM II, and the second is to validate the correction methods for this effect. To achieve these goals, the MISOLFA instrument measures all the optical parameters, which allows the observation conditions in which the measurements are made to be quantified. These parameters are the Fried parameter $r_{0}$, the spatial coherence outer scale $L_{0}$, the isoplanetism domain $\theta_{0}$, the temporal characteristic(s) of the wave front evolution $\tau_{0}$, and the turbulence vertical profile $C_{n}^{2}(h)$. To perform solar radius measurements, the chosen wavelengths are the same as that of the SODISM II instrument (393, 535, 607, 782, and $1025 \mathrm{~nm})$. Currently, only one wavelength is operational $(535 \mathrm{~nm}$ with $\Delta \lambda=2.5 \mathrm{~nm})$. The exposure time of SODISM II images at $535.7 \mathrm{~nm}$ (a) is $1.3 \mathrm{~s}$. The mean recorded turbulence characteristic time $\left(\tau_{0}\right)$ is about $20 \mathrm{~ms}$, therefore SODISM II images can be considered as long-exposure times (see Fig. 5).

\subsection{The PAPS photometer and aerosols detection}

Details about liquid and solid aerosols, especially cirrus, subvisible cirrus, and generally transparency of the atmosphere above the observation site are required to properly correct the SODISM II observations. Aerosol influence on solar radius measurements has motivated our interest. Indeed, all the atmospheric effects may have an impact on our measurements. Thus, an automatic photometer (Photomètre Automatique Picard Sol or PAPS) provides a quality index for the pictures taken by SODISM II. The main purpose of PAPS is measuring Sun and sky radiance to derive the total column water vapor $(\mathrm{cm})$, ozone, and aerosol properties using a combination of spectral filters. This instrument is included in the aerosol robotic network (AERONET) program (Holben et al. 2001). Aerosol optical thickness (AOT) is a quantitative measurement of the extinction of solar radiation by aerosol scattering and absorption between the point of observation and the top of the atmosphere. AOT can be determined from the ground through measurements of the spectral transmission of solar radiation through the atmosphere using the Picard Sol photometer. To perform solar radius measurements, the wavelengths $(340 \mathrm{~nm}, 380 \mathrm{~nm}, 440 \mathrm{~nm}, 500 \mathrm{~nm}, 675 \mathrm{~nm}, 870 \mathrm{~nm}$, and $1020 \mathrm{~nm}$ ) are chosen very close to those used by the SODISM II instrument. Diffusion by aerosols affects image contrasts, and Fig. 7 shows the effect of different atmospheric conditions on two adjacent days on the limb-shape and its first derivative. The interpretation of Fig. 7 in terms of the influence of aerosols alone is not possible because the optical turbulence in the two cases is not necessarily the same. Simulations show that the decrease of contrast does not lead to significant bias on the location of the inflection point. It affects the precision of the measurement, however.

\subsection{The PPS pyranometer and solar radiation monitoring}

The Picard Sol pyranometer (Pyranomètre Picard Sol or PPS) is a radiometer that measures on a flat surface the sum of the luminous flux received from the Sun and the scattered solar light by the atmosphere after many reflections between the ground and atmosphere particles (molecules and aerosols). In clear atmospheric conditions, the received energy has a distribution as a function of time (shape with maximum at local solar noon). If there are clouds or more generally aerosols, this distribution departs from the previous shape by a decrease of the energy received by the instrument. This is seen by comparing the received energy distribution measured on an adjacent clear day. However, there are cases of enhanced solar irradiance at ground level that occur when there are high altitude cloud such as altocumulus and cirrus. This instrument detects this effect by an increase of the received energy. Figure 8 shows such an occurrence. The pyranometer detects these circumstances, but integrates the whole sky and therefore does not provide the relevant information in the direction of the Sun alone. An automatic determination of cloud type is possible with these data (Duchon \& O'Malley 1999), however, which would be interesting to combine in a future work with the information on aerosols provided by PAPS.

\subsection{The CPS visible wide-field camera and nature of the detected clouds}

A Picard Sol visible wide-field camera (Caméra Picard Sol or CPS), operating in color (SBIG AllSky-340, Santa Barbara Instrument Group) is used to detect the location of clouds, which the pyranometer and photometer do not allow. This instrument permits detecting thin cirrus because of its great sensitivity and provides complementary information to the pyranometer and the photometer (aerosols). CPS brings us information about the quality of the sky (Fig. 9) and high-altitude clouds.

\section{Picard Sol instrumental calibrations and operations}

\subsection{Picard Sol instrumental calibrations}

\subsubsection{Angular calibration for the SODISM II plate-scale determination}

The knowledge of the SODISM II plate-scale is a fundamental parameter to derive an absolute value of the solar radius. Moreover, to discuss its wavelength dependence, we also need to obtain the plate-scale at different wavelengths. This can first be estimated theoretically using the appropriate optical 
M. Meftah et al.: Ground-based measurements of the solar diameter during the rising phase of solar cycle 24
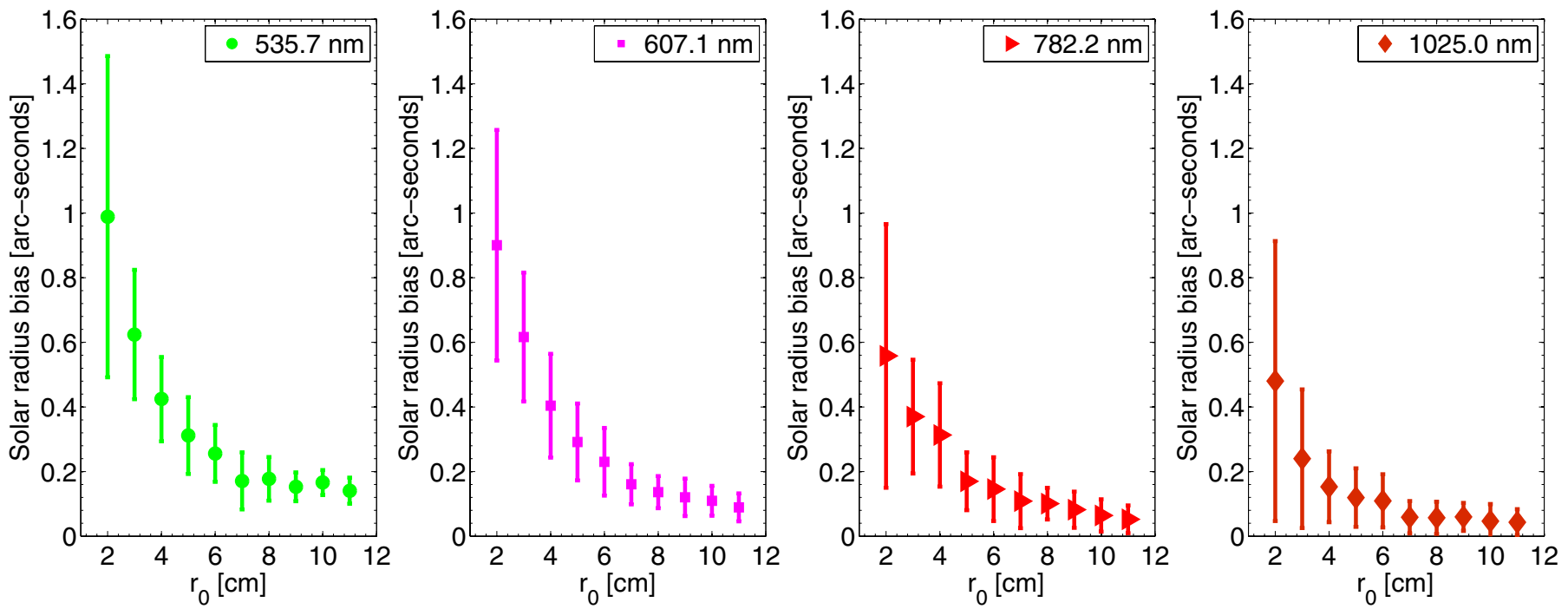

Fig. 5. Simulation of the bias due to Fried parameter $\left(r_{0}\right)$ on SODISM II measurements (Von Kàrmàn model) with long-exposure times $\left(50 \times \tau_{0}\right)$.
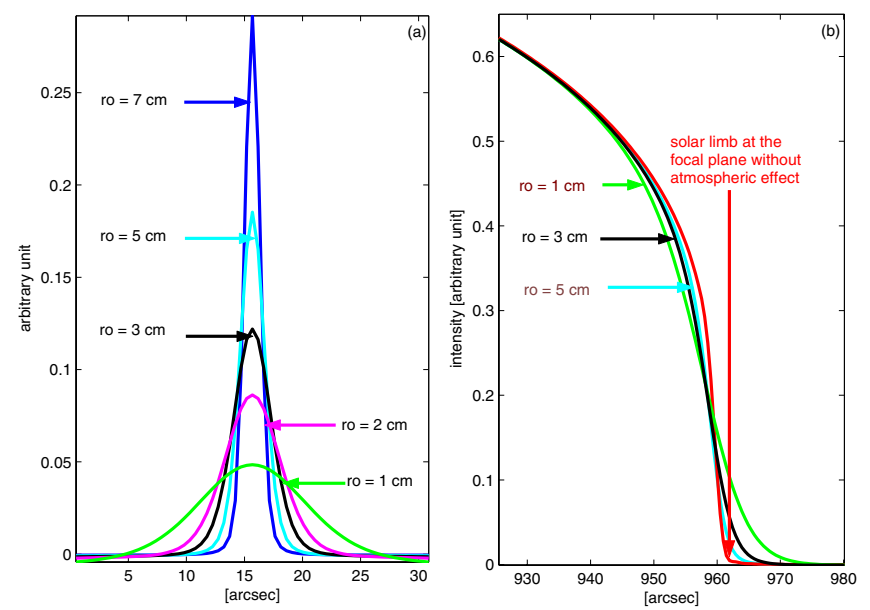

Fig. 6. Effect of turbulence on the limb-shape b) and its derivative a) as a function of $r_{0}$.

configuration (optical thickness of the different filters, point spread function (PSF), etc.). However, given the level of accuracy required for our measurements, a specific experimental calibration is also required. This is achieved by observing pairs of stars selected according to their brightness and angular distances, which must be close to 30 arcmin. The highest acceptable magnitude is 5 and the elevation must be greater than 20 arcdegrees given the limitation of the equatorial mount and to avoid strong atmospheric refraction. Five pairs of stars have been selected for measurements at different periods of the year (Meftah et al. 2012). Data processing includes three types of corrections:

1. proper motion of the star, parallax depending on the position of Earth around the Sun, and aberration;

2. chromatic effects, because the measurements are made without interference filter using stars of different types to infer the plate-scale conversion at the wavelength of the SODISM II measurements through an interference filter of known optical thickness;

3. thermal effects, because the measurements are made in the night-time. Thermal corrections to the focus applied for night-time star observing are weak. Indeed, our front window is inside the vacuum tank, which is controlled in temperature.
Another way for estimating SODISM II plate-scale is to use the solar radius obtained by the SODISM space instrument during the Venus transit as reference (Meftah et al. 2014a). The synthesis associated with these three approaches is given in Table 3. Two experimental calibrations (ACPS and SRPS) lead to results that agree at a level better than $5 \times 10^{-5}$. These results and the wavelength dependence are strongly linked to the knowledge and proper modeling of the instrumental optical configuration, however. In the following, we adopt the SRPS values, with which we associate conservative uncertainties of $10^{-4}$. This introduces an uncertainty of \pm 0.090 arcsec on the determination of the absolute value of the solar radius.

\subsubsection{SODISM II radiometric calibrations}

The image data of the SODISM II solar telescope require dark-signal corrections (Hochedez et al. 2014). SODISM II dark-current images have been performed every day and with an exposure time of $1.4 \mathrm{~s}$. All images have been corrected for dark-current.

Our images also require flat-field corrections, which consist of measurements allowing to set the pixels responsivity on the same photometric scale. This is potentially important for the precise determination of the limb-shape from which the solar radius is obtained. The whole SODISM II system must be used, including the interference filters. For flat-field measurements, the absence of clouds is mandatory, which is ensured by taking into account the observations gathered by the PAPS photometer, the PPS pyranometer, and the CPS camera. We used the method developed by Kuhn et al. (1991), which is based on the displacement of multiple images from the same source, here, the Sun. The set of images covers the CCD by scanning the Sun using the solar mounting. During the displacement, the Sun irradiance constancy is checked by using the pyranometer data and the integrated intensity within the image. As expected, operation made around solar noon is preferable. For each wavelength, the duration of the operation is around 80 minutes for acquiring 64 images. It is difficult to ensure that we keep excellent and stable conditions over such a long period and therefore it is very difficult to obtain a good flat-field on a regular basis for all wavelengths this way. A new method based on contrast maps has been developed and is being tested. This method basically assumes that the limb darkening function (LDF) is theoretically 

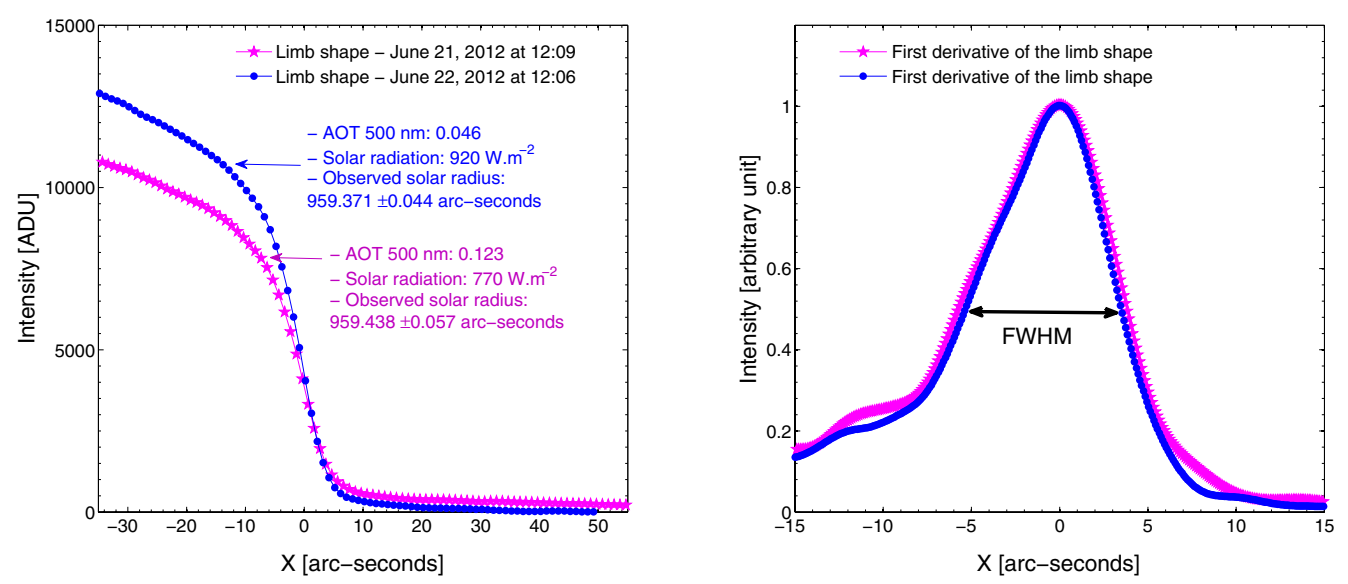

Fig. 7. Left: atmospheric effect on limb-shape at $535.7 \mathrm{~nm}$ (b). Right: evolution of the limb-shape first derivative. FWHM of the solar limb first derivative decreases as AOT decreases.

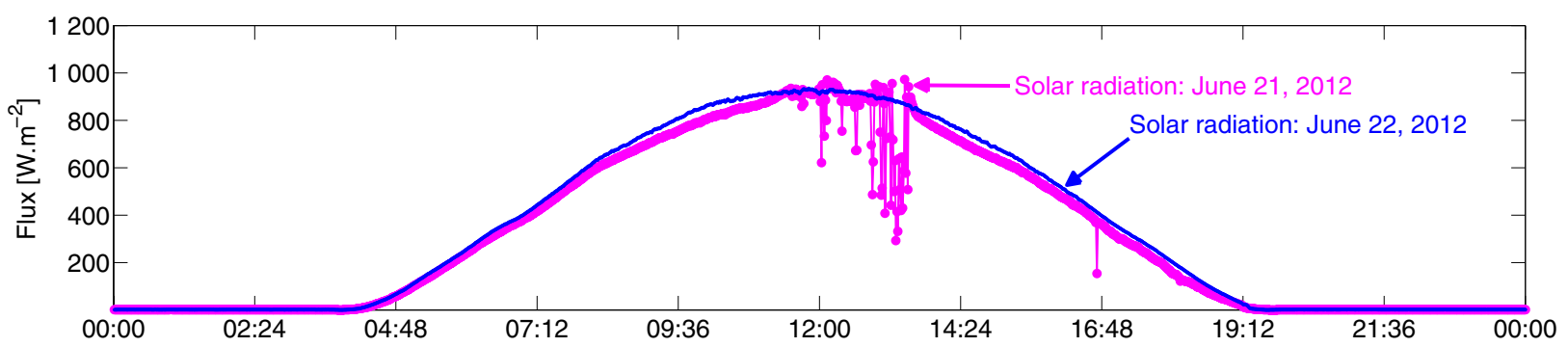

Fig. 8. Superposed solar radiation received on an horizontal plane in clear and slightly perturbed atmospheric conditions. The blue curve shows the measurement on the next day to allow a comparison with clear air conditions.

Table 3. SODISM II plate-scale for different wavelengths.

\begin{tabular}{lccccc}
\hline \hline Wavelength [nm] & Optical thickness [mm] & TPS & ACPS & SRPS & SRPS-ACPS \\
\hline 393.37 & 12.328 & 1.060818 & 1.061076 & 1.061102 & $2.63 \times 10^{-5}$ \\
535.7 (a) & 12.297 & 1.060792 & 1.061057 & 1.061060 & $2.75 \times 10^{-6}$ \\
535.7 (b) & 12.303 & 1.060796 & 1.061061 & 1.061064 & $2.92 \times 10^{-6}$ \\
607.1 & 12.225 & 1.060757 & 1.061016 & 1.061025 & $9.42 \times 10^{-6}$ \\
782.2 & 12.341 & 1.060781 & 1.061083 & 1.061049 & $-3.40 \times 10^{-5}$ \\
1025.0 & 12.080 & 1.060679 & 1.060931 & 1.060947 & $1.54 \times 10^{-5}$ \\
\hline
\end{tabular}

Notes. The optical thickness column corresponds to a characterization of the different filters. SODISM II interference filters do not have the same optical thickness (index, thickness of optical elements used, etc.). Thus, each wavelength has its own plate-scale correction factor. Theoretical plate-scales (TPS in arcsec pixel $^{-1}$ ) are obtained from an optical model using the appropriate optical configuration. Angular calibration platescales (ACPS in arcsec pixel ${ }^{-1}$ ) are obtained during specific campaigns on star doublets. SODISM reference plate-scales (SRPS in arcsec pixel ${ }^{-1}$ ) are obtained from the knowledge of the solar radius measured by SODISM at $607.1 \mathrm{~nm}$ during the Venus transit. Six digits of the TPS, ACPS, and SRPS entries are significant.
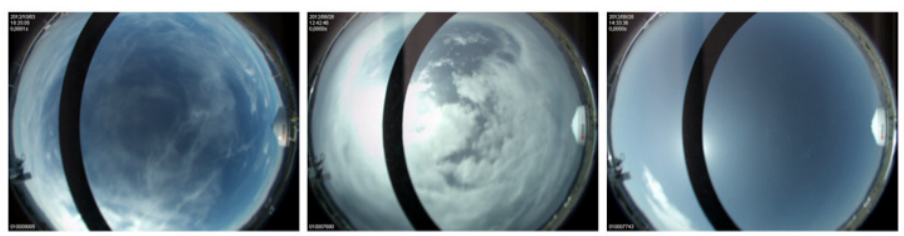

Fig. 9. Three typical situations encountered during operations that show different atmosphere transparency. They provide additional information for the instrumental calibration.

well known at least up to 0.8 solar radius and can be removed from each image after a proper normalization. The resulting contrast map is then assimilated to a uniformly illuminated plane, and the imperfect tracking of the Sun center during an observation sequence is used to build the flat-field. The effects of active regions are eliminated by taking for each pixel a median value over the whole sequence of contrast maps (Fig. 4). Our first tests have shown that the effect of flat-field corrections on the determination of the mean inflection point location is lower than 1 mas.

\subsection{Picard Sol operations}

The seeing monitor MISOLFA and the solar imager SODISM II have observed the Sun together since March 2011. SODISM II records full solar images at several wavelengths, with an optimal cadence of one image per minute (nominal operations since May 6, 2011). At the same time, MISOLFA records a continuous sequence of high-resolution ( $0.2 \mathrm{arcsec})$ and high-cadence (32 images per second) limb images at $535 \mathrm{~nm}$ from the two opposite sides of the solar disk as well as the temporal irradiance fluctuations on the pupil channel. 

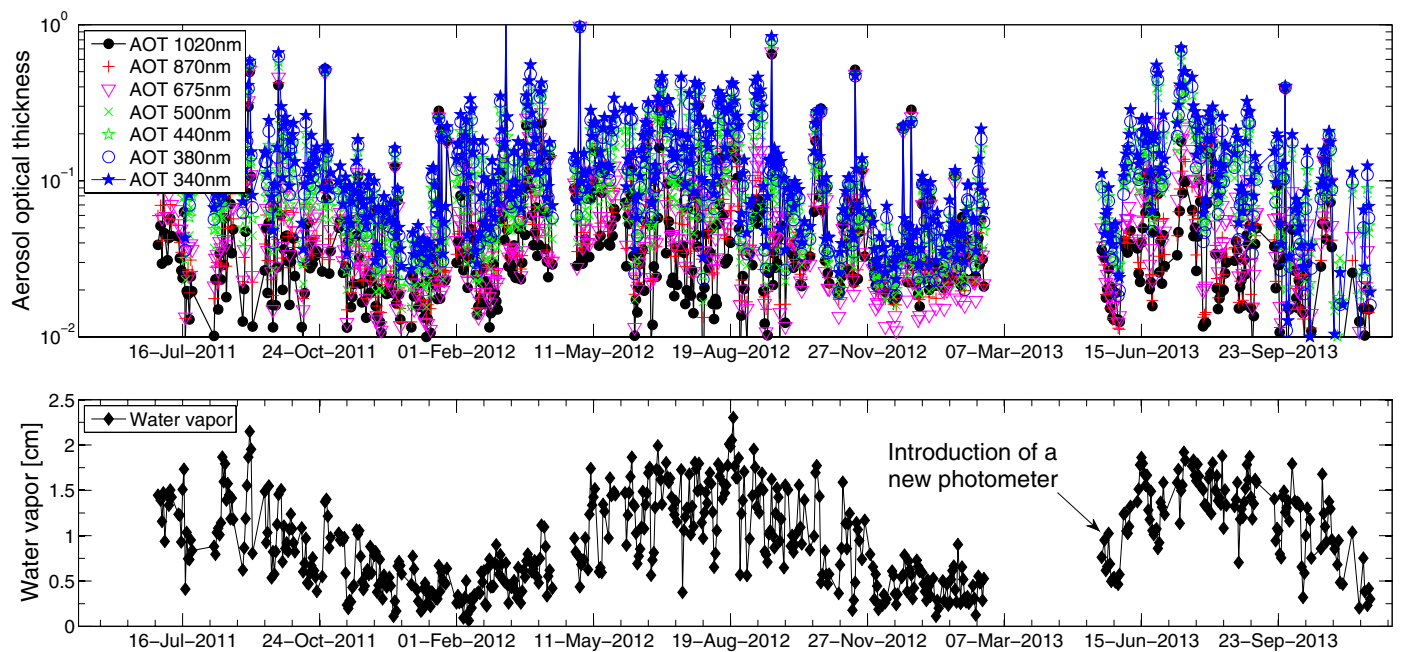

Fig. 10. Aerosols optical thickness (AOT) and water vapor content as a function of time at the observation site of Calern. The measurements obtained are part of data selection process.

\section{Data selection, SODISM II corrections, and uncertainty budget}

\subsection{SODISM II data selection}

The main causes of perturbation acting on the solar images are the transparency of the atmosphere and turbulence. The transparency of the atmosphere is of prime importance for the quality of the measurements. Scattered light has an effect on the image contrast and the limb-shape. Correcting for it would be difficult even if the detailed structures of the aerosols cloud (solid or liquid) were known. The best way consists of performing a drastic data selection. To achieve it, we dispose of key information about the aerosols load and presence of cirrus, which is more frequent in summer (constituting June, July, and August) than in winter (December, January, and February). The additional instrumentation (photometer, pyranometer and camera) provides a quality index for solar images taken by SODISM II and allows us to reject all data that are contaminated by atmospheric effects. Figure 10 shows that in terms of atmospheric aerosol, local winter is preferable. However, the lower mean Sun elevation in winter corresponds to a higher air mass, which also affects the mean image contrast. This is why both summer and winter images can pass the data selection process. The only way to minimize the presence of aerosol or to reduce its seasonal variations would be to observe from an observatory at a higher altitude.

\subsection{SODISM II corrections}

\subsubsection{SODISM II correction for Sun-Earth distance (Calern-Sun distance)}

The average Sun-Earth distance is called the astronomical unit (1 AU is equal to $149597870.700 \mathrm{~km}$ ). It is a simple matter to correct the solar radius measurements within any time to their corresponding values at $1 \mathrm{AU}$ based on the well-known ephemeris (Calern observatory). An error of one minute on dating images (impact on ephemeris value) would correspond to a maximum of 1 mas uncertainty on the determination of the solar radius.

\subsubsection{Refraction and SODISM II computational method}

Effect of refraction on the mean solar radius is corrected. The key inputs for this correction are temperature $(T)$, pressure $(P)$, and relative humidity $\left(f_{\mathrm{h}}\right)$ locally measured with the additional instrumentation. The standard conditions for Calern site are: (i) $T=15^{\circ} \mathrm{C}$; (ii) $P=875 \mathrm{hPa}$; and (iii) $f_{\mathrm{h}}=50 \%$. For temperature, pressure, and relative humidity, we assumed uncertainties of $\Delta T=0.5{ }^{\circ} \mathrm{C}, \Delta P=1 \mathrm{hPa}$, and $\Delta f_{\mathrm{h}}=5 \%$, which are typical for a standard weather station. Thus, the uncertainty on the correction is smaller than 20 mas for zenith distances lower than $70^{\circ}$. In this work, the correction for refraction is directly applied on the mean solar radius. For more accurate measurements, the corrections can be applied individually for each heliographic angle. This method should be used in future work to distinguish and understand the different effects on solar radius measurements (optical aberrations, turbulence, etc.).

\subsubsection{Turbulence and MISOLFA measurements}

The main source of uncertainty and bias for any groundbased measurement is certainly the effect of optical turbulence. Attempts to reduce this effect were historically made by using the finite Fourier transform definition of an edge on the solar disk (Brown 1982). This definition of the solar edge is based on a filtered integral of the solar signal at the solar limb. This minimizes the influence of the varying slope of the observed LDF that is induced mostly (but not only) by the varying atmospheric conditions such as seeing and scattering. Thus, the effect of turbulence is a spreading of the limb-shape that leads to a decrease of the solar radius. In other terms, turbulence generates a biased solar radius measurement. This bias can be estimated by using a model of turbulence and a given limb-shape. The results are shown in Fig. 5 using the model of Von Kàrmàn (Ikhlef et al. 2012). The bias on solar radius is decreasing with increasing $r_{0}$ and reaches an asymptotic value that depends on the wavelength (for solar continuum). As we show below, $r_{0}$ found in our observations is around $3 \mathrm{~cm}$ (at $535 \mathrm{~nm}$ ), which implies a bias correction of about 0.6 arcsec, with a root mean square (RMS) of about 0.2 arcsec (at $535.7 \mathrm{~nm}$ ). MISOLFA and SODISM II are simultaneously operated, which allows us, in principle, to correct each solar radius. The detailed treatment of MISOLFA records is still underway, however, and in this preliminary work, we use 
Table 4. Fried parameter measurement performed between June 2010 and May 2012 (monthly median values $r_{0}$ and standard deviation $\sigma$ ).

\begin{tabular}{lcccccccccccc}
\hline \hline Month & Jan. & Feb. & Mar. & Apr. & May & Jun. & Jul. & Aug. & Sep. & Oct. & Nov. & Dec. \\
\hline$r_{0}[\mathrm{~cm}]$ & 2.82 & 3.42 & 2.92 & 3.71 & 4.10 & 4.20 & 3.88 & 2.50 & 3.70 & 3.45 & 3.50 & 2.75 \\
$\sigma[\mathrm{cm}]$ & 0.94 & 1.26 & 1.11 & 1.28 & 1.32 & 1.32 & 1.76 & 1.16 & 1.24 & 1.19 & 0.91 & 0.50 \\
\hline
\end{tabular}
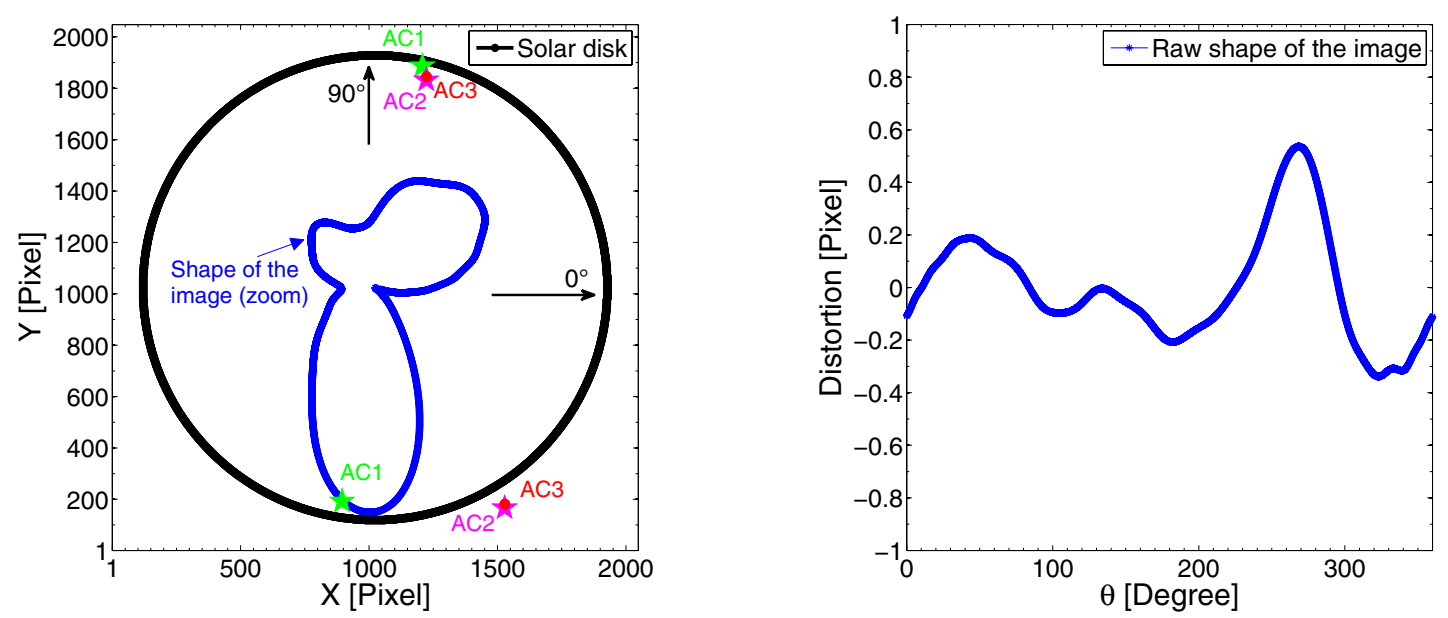

Fig. 11. Left: the solar disk as seen on the CCD shown with the black curve. The raw shape of the SODISM II image (solar radius at a given angle minus its lowest value) is represented with the blue curve. The scale is amplified by a factor of 1000. The three stellar angular calibrations (AC) that have been performed are represented by star symbols. The SODISM II plate-scale also depends on the shape of the image. Right: average limb position vs. central angle plotted in pixel units. The shape of the image corresponds to a SODISM II optical default (astigmatism). This shape does not change significantly over time (few mas).

only an average value of $r_{0}$. In a future work, the use of monthly averages of the Fried parameter should remove most of the seasonal effects induced by turbulence, but the ultimate goal with MISOLFA is to obtain reliable estimates of $r_{0}$ every minute. The Fried monthly averaged parameter measured from 2010 to 2012 is shown in Table 4.

\subsubsection{SODISM II distortion and shape of the raw image}

We defined the solar radius by the inflection-point position (IPP) of the solar-limb profiles taken at different angular positions $(\theta)$ of the image. The IPP is obtained by the passage through zero of the solar limb second derivative. The shape of SODISM II raw image (contour) is obtained from the calculation of all inflection points. Figure 11 shows the raw shape of SODISM II images obtained at low zenith distance to avoid astronomical refraction effects. The shape of the image is at most one pixel (around one arcsec). Knowledge of the image shape is critical for different calibrations (plate-scale) and corrections. Measurements of solar oblateness are not achievable with SODISM II instrument. It has been established from space missions that the difference in equator-to-pole radius is smaller than $10^{-2}$ arcsec (Kuhn et al. 2012). Thus, we can consider that the shape of the Sun is a perfect solar disk. Therefore, the shape of the image depends on the refraction, turbulence, and optical aberrations. In Fig. 11, the positions of three pairs of stars used for the plate-scale calibration are shown. The values of the plate-scale deduced from the measurements of their angular distance on the CCD were corrected using the information on image distortion provided by the observed shape of the solar disk.

\subsubsection{SODISMII PSF}

The SODISM II PSF and its effect on the solar limb were studied for the nominal optical configuration (Fig. 12), wherein the instrument is diffraction limited. Indeed, the SODISM II design and dimensions are such that the size of the Airy diffraction disk is about one arcsecond in the bluest case, which is still larger than any of the expected aberrations. The LDF of HM98 (Hestroffer \& Magnan 1998), the COSI code for solar irradiance (Haberreiter et al. 2008a; Shapiro et al. 2010) and 3D hydrodynamic and magneto-hydrodynamic simulations or 3D model (Piau et al. 2011) were convolved by the theoretical PSF of the telescope, and the results for each wavelength are represented in Fig. 12 (LDF, PSF, and first derivative of the LDF convolved with the instrument PSF). The first derivative of the limb is spread over about two arcsec, and the location of the inflection point (maximum of the first derivative) appears to be clearly defined for all LDF models. We then studied the quality of the SODISM II images to see whether a solar radius measurement can be achieved with those data. We show in Fig. 7 (left panel) the solar limb intensity darkening extracted from solar images recorded at $535.7 \mathrm{~nm}$ (b) in two typical cases. We next compute the first derivative of these two LDFs to better evaluate their spread (right panel in Fig. 7). The observational curve gives an indication of image quality. The first derivative of the solar limb recorded with SODISM II is significantly wider than the spread expected from the model (Fig. 12, bottom panel at $535.7 \mathrm{~nm}$ ). The slope of the observed intensity profile - defined as the full width at half maximum of its first derivative is wider than expected by $\sim 6$ arcsec. This might be caused by a misalignment of the optical elements (astigmatism), combined with turbulence effects, and thermo-optical effects, which can also blur the image. In itself, this does not disqualify the scientific objective since it is equivalent to having a telescope with a smaller aperture, giving the observed PSF. But it would have to be time invariant that is constant during the entire mission. Thus, we do not seek to obtain the best focus. We simply wish to have a stable instrument. The daily means of observed LDF first derivative FWHM (Fig. 13) show a slight seasonal modulation, 
M. Meftah et al.: Ground-based measurements of the solar diameter during the rising phase of solar cycle 24
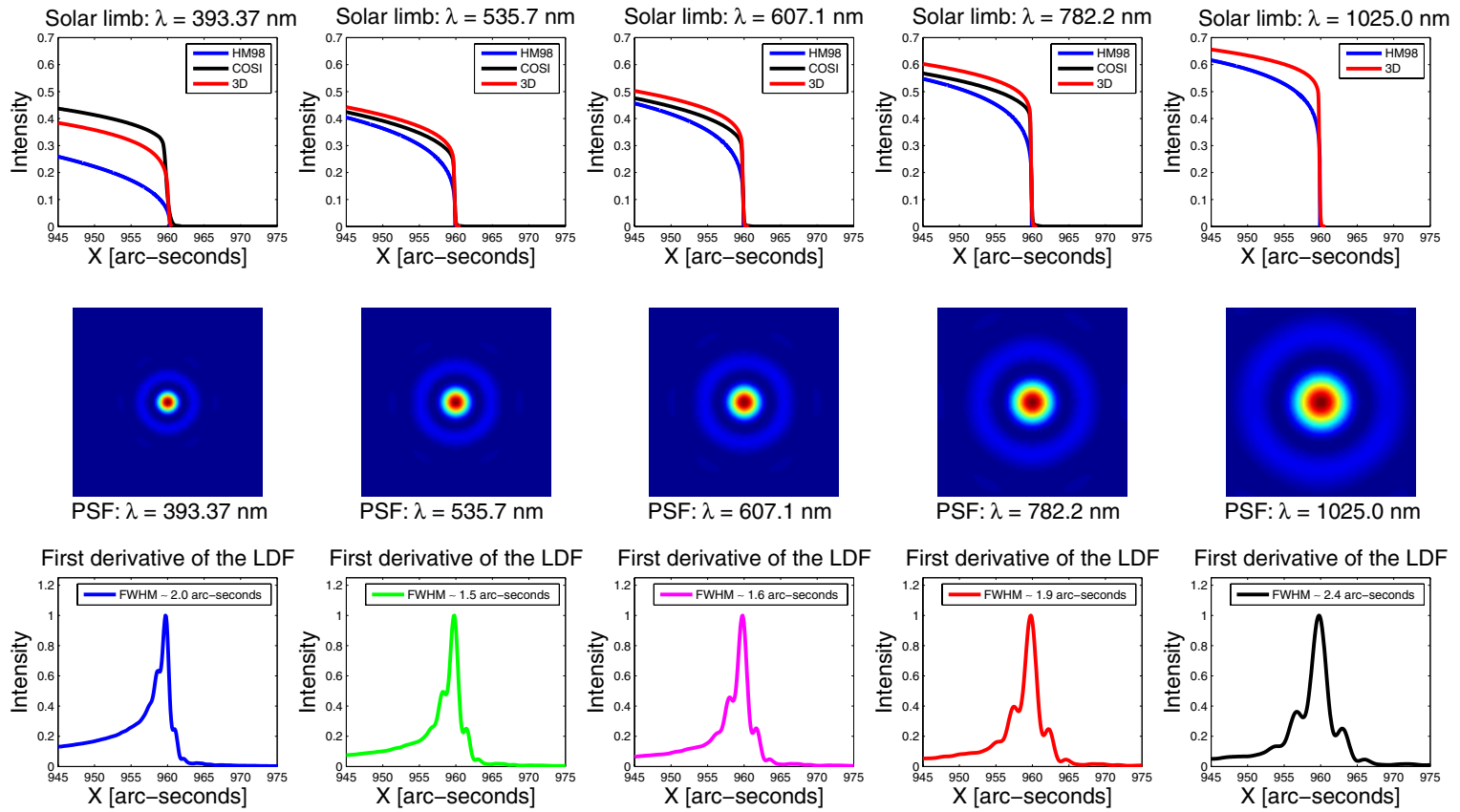

Fig. 12. Top: LDF at 393.37, 535.7, 607.1, 782.2 and $1025.0 \mathrm{~nm}$, for the HM98 (empirical power-law model extrapolated to the limb), COSI models and 3D model. Their convolution with the nominal PSF of SODISM II is dominated by diffraction. Middle: SODISM II PSF for each wavelength. These PSF have been estimated by an optical model of SODISM II. Bottom: first derivative of the convolution between the two observables (LDF and PSF). It can be verified visually that the FWHM of the first derivative of the solar limb is about two arcsec.

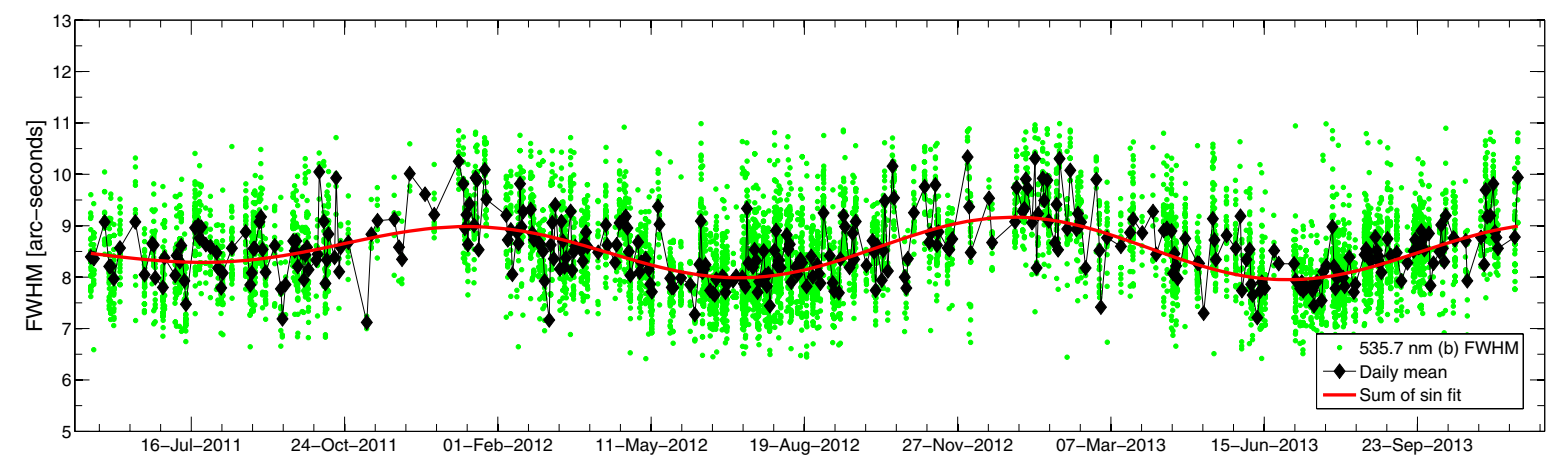

Fig. 13. Evolution of the solar limb first derivative FWHM at $535.7 \mathrm{~nm}$ (b) since the beginning of the mission (green dot). The black curve with diamond symbols corresponds to the FWHM of the daily mean at $535.7 \mathrm{~nm}$ (b).

however, which is probably related to the seasonal variations of the mean elevation of the Sun in the sky, which modulates both the influence of turbulence and the mean image contrast.

\subsection{SODISM II uncertainty budget for determining the solar radius}

Uncertainties for the absolute accuracy of the solar radius for different wavelengths are given in Table 5. Turbulence is a major source of uncertainty. The spatial and temporal variations of the refractive index change the path of light as it travels through the atmosphere, causing image motion and blurring. Turbulence limits the resolving power of the telescope. The Fried parameter, which qualifies the observations (seeing), is the dominant element in calculating the phase fluctuation variance. Considering a Kolmogorov model, the Fried parameter $\left(r_{0}\right)$ is related to the angle of arrival fluctuation variance $\left(\sigma_{\alpha}^{2}\right)$ by

$r_{0}=8.25 \times 10^{5} \times D^{-\frac{1}{5}} \times \lambda^{\frac{6}{5}} \times\left(\sigma_{\alpha}^{2}\right)^{-\frac{3}{5}}$, where $D$ is the aperture of the telescope $(\mathrm{m})$ and $\lambda$ is the wavelength $(\mathrm{m})$. This equation was used to compute the Fried parameter, and some results shown in the Table 4. It is predicted from the Kolmogorov theory of turbulence, and the known dispersion of air, that the Fried parameter varies with wavelength as $r_{0} \infty \lambda^{\frac{6}{5}}$. As mentioned above, the average Fried parameter $r_{0}$ is $3.41 \mathrm{~cm}$ at $535 \mathrm{~nm}$. Thus, from the proportional relationship, we can determine the different values of $r_{0}$ for SODISM II different wavelengths $(3.97 \mathrm{~cm}$ at $607.1 \mathrm{~nm}, 5.38 \mathrm{~cm}$ at $782.2 \mathrm{~nm}$ and $7.44 \mathrm{~cm}$ at $1025.0 \mathrm{~nm}$ ). Therefore, we know the bias and the associated uncertainty on solar radius measurement for each wavelength $(540 \pm 170$ mas at $535.7 \mathrm{~nm}, 410 \pm 160$ mas at $607.1 \mathrm{~nm}, 160 \pm 90 \mathrm{mas}$ at $782.2 \mathrm{~nm}$ and $60 \pm 50 \mathrm{mas}$ at $1025.0 \mathrm{~nm}$ ), and that from Fig. 5.

The SODISM II instrument is thermally controlled and appears to be robust against changes on the order of few microns or a few degrees of temperature (Meftah et al. 2014b). Temperatures of the instrument (housekeeping) are measured, and their variations (thermal effects) introduce an uncertainty 
Table 5. SODISM II uncertainties for all wavelengths.

\begin{tabular}{lccccc}
\hline \hline Uncertainty sources & 535.7 (a) & 607.1 & 782.2 & 1025.0 & Type \\
\hline Angular calibration & 90 & 90 & 90 & 90 & Random \\
Aerosols & - & - & - & - & $\mathrm{D}^{a}$ \\
Calern-Sun distance & $\leq 1$ & $\leq 1$ & $\leq 1$ & $\leq 1$ & Random \\
Refraction & $\leq 20$ & $\leq 20$ & $\leq 20$ & $\leq 20$ & Random \\
Turbulence & 170 & 160 & 90 & 50 & Random \\
Optical distortion & - & - & - & - & $\mathrm{C}^{c}$ \\
PSF & - & - & - & - & $\mathrm{C}^{c}$ \\
Scattered light & - & - & - & - & $\mathrm{N}^{b}$ \\
Pointing & - & - & - & - & $\mathrm{D}^{a}$ \\
Thermal effects & $\leq 10$ & $\leq 10$ & $\leq 10$ & $\leq 10$ & Random $^{\text {dindom }}$ \\
\hline
\end{tabular}

Notes. All values are in mas. ${ }^{(a)}$ D: Data selection. ${ }^{(b)} \mathrm{N}$ : Negligible. ${ }^{(c)}$ C: Calibration.

smaller than 10 mas on the determination of the solar radius (for each wavelength).

\section{Results and discussion}

\subsection{Solar radius determination}

\subsubsection{Mean solar radii obtained after refraction correction}

An evolution of the solar radius measurements obtained with SODISM II (SRPS reference) is shown in Fig. 14 as a function of time for different wavelengths and after refraction correction. From these corrected measurements, a mean solar radius for each wavelength is given in Table 6. The SODISM II mean radius of $959.237 \operatorname{arcsec}$ (with $\sigma=0.25 \mathrm{arcsec}$ ) at $535.7 \mathrm{~nm}$ (a) is deduced from 20300 measurements. This is our reference time-series (comparison with historical measurements made at Calern). The various evolutions indicate that with increasing wavelength (solar continuum) the mean solar radius increases. At $393.37 \mathrm{~nm}$, chromospheric emissions are recorded. Given its location above the photosphere, the solar radius is larger than those measured by using photospheric emissions. There is a difference of 700 mas compared with our reference wavelength. Without turbulence and with a perfect instrument, this difference must be on the order of 400 mas. This channel requires a special analysis.

\subsubsection{Mean solar radii obtained after turbulence correction}

The final SODISM II results (solar continuum), after making all necessary corrections described in the previous sections, are listed in the Table 7. The value of the solar radius is found to be equal to $959.777 \mathrm{arcsec}$ at $535.7 \mathrm{~nm}$ (a), while the estimated uncertainties of the measurements are typically smaller than 0.2 arcsec. At $1025.0 \mathrm{~nm}$, the solar radius is smaller than those found at $607.1 \mathrm{~nm}$ and $782.2 \mathrm{~nm}$.

\subsection{Solar radius variability}

The current solar cycle is probably going to be the weakest in 100 years, which is an unprecedented opportunity for studying the variability of the solar radius during this period. The SODISM II measurements we made are reproducible. We did not observe any significant degradation. The solar radius observed with SODISM II results from variations smaller than 50 mas that are out of phase with solar activity. Solar radius variations for SODISM II and different instruments are plotted in Fig. 2,
Table 6. SODISM II mean solar radius (R) for each wavelength and standard deviation $(S$ at $1 \sigma)$ of the daily mean values after refraction correction.

\begin{tabular}{lcccc}
\hline \hline Wavelength [nm] & $N$ & $d$ & $R$ & $S$ \\
\hline 393.37 & 11309 & 367 & $959.940^{\prime \prime}$ & $0.106^{\prime \prime}$ \\
535.7 (a) & 20300 & 374 & $959.237^{\prime \prime}$ & $0.125^{\prime \prime}$ \\
535.7 (b) & 11391 & 376 & $959.286^{\prime \prime}$ & $0.103^{\prime \prime}$ \\
607.1 & 11342 & 350 & $959.452^{\prime \prime}$ & $0.126^{\prime \prime}$ \\
782.2 & 11351 & 375 & $959.716^{\prime \prime}$ & $0.099^{\prime \prime}$ \\
1025.0 & 11759 & 375 & $959.772^{\prime \prime}$ & $0.181^{\prime \prime}$ \\
\hline
\end{tabular}

Notes. $N$ is the total number of measurements gathered between May 2011 and December 2013, and $d$ is the number of observation days.

showing inconsistent results. Nevertheless, we can observe the measurement continuity between SODISM II and MDI.

\subsection{Spectral dependence of solar continuum radius}

One of the objectives of the PICARD mission is to deduce the spectral dependence of the solar limb profile. A study of the shape of the solar limb (models and observations) was carried out by Thuillier et al. (2011). Several kinds of solar atmosphere models were compared. Some models are constructed empirically for instance that of HM98, but contain no IPP. Others use theoretical models, such as VAL81 (Vernazza et al. 1981), FCH09 (Fontenla et al. 2009), and COSI, or are based on physical principles, such as SH09 (Short \& Hauschildt 2009). Finally, others use 3D numerical simulations. Table 8 compares the IPP for four wavelengths. The different solar models show the same trend and their predictions agree well. The differences $(\Delta \mathrm{IPP})$ of the inflection-point position at $607.1 \mathrm{~nm}, 782.2 \mathrm{~nm}$ and $1025.0 \mathrm{~nm}$ from the IPP reference at $535.7 \mathrm{~nm}$ are smaller than thirty mas for the different models, but numerous studies of solar radius variation at different wavelengths appear in the literature that show a higher wavelength dependence, but with conflicting results. These measurement differences are most likely dominated by a poor correction of the wavelength dependence of instrumental (plate-scale, PSF, etc.) and atmospheric effects and not on the weak wavelength dependence of the solar LDF itself in the photospheric continuum.

From a theoretical model, we analyzed the impact of an instrumental effect on the inflection-point position. Figure 15 highlights the impact of an optical aberration (astigmatism) on our fine metrology measurements. The trend we see with the instrument is real. Indeed, three interference filters are from the same fabrication batch $(535.7,607.1$, and $782.2 \mathrm{~nm})$. A more detailed laboratory measurement of the instrument PSF can help us to refine this result. At $1025.0 \mathrm{~nm}$, the result is more delicate. For this wavelength, we manufactured the interference filter after all others. There may be a low deviation in the determination of the optical thickness. Moreover, for this wavelength the quantum efficiency of the CCD is poor and the difference between the mean corrections introduced for turbulence (Table 7) is 100 mas between $782.2 \mathrm{~nm}$ and $1025.0 \mathrm{~nm}$. This is based on the theoretical $\lambda^{6 / 5}$ dependence of $r_{0}$, but needs to be confirmed experimentally. 


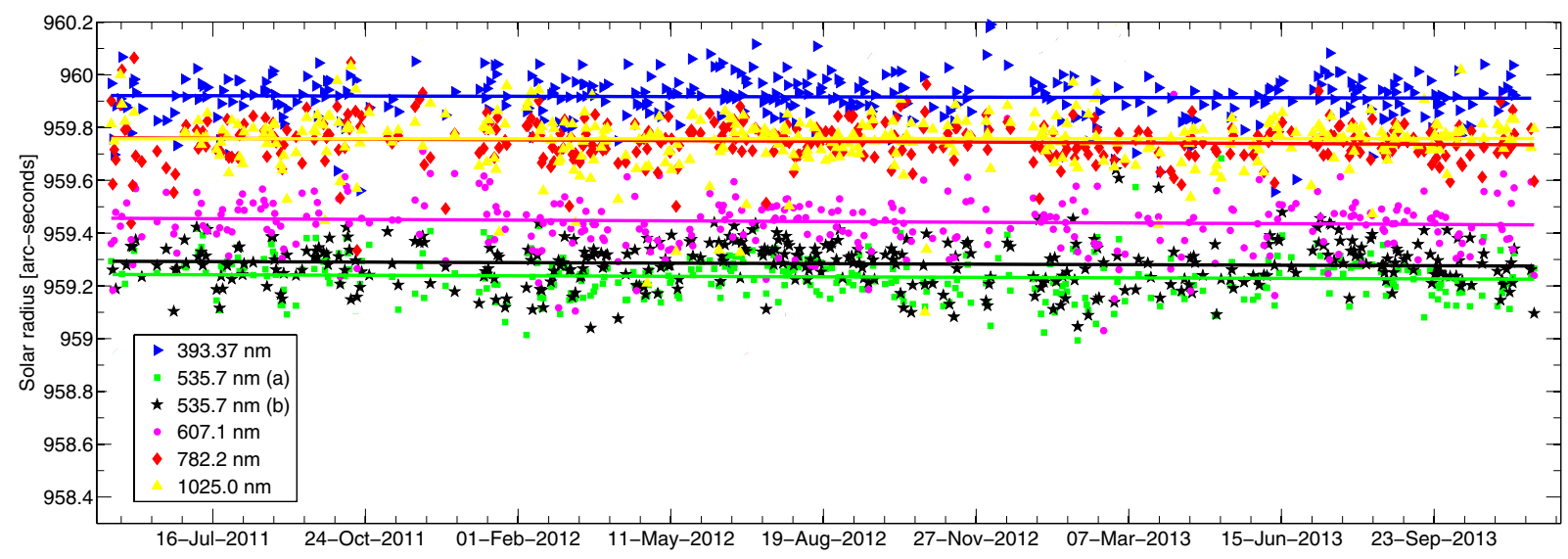

Fig. 14. Daily mean SODISM II observed solar radius with refraction correction as a function of time for different wavelengths. For all wavelengths, we obtained a negative slope of about 12 mas per year, which is not significant when compared with the $1 \sigma$ uncertainty obtained on the mean radius (between 100 and 200 mas in Table 6). Any long-term instrumental degradation of the PSF or focus would lead to a slightly lower radius estimate.

Table 7. SODISM II mean solar radii and associated combined standard uncertainties after turbulence correction.

\begin{tabular}{lcccc}
\hline \hline Wavelength $[\mathrm{nm}]$ & $535.7(\mathrm{a})$ & 607.1 & 782.2 & 1025.0 \\
\hline Radius $\left.{ }^{a}{ }{ }^{\prime \prime}\right]$ & 959.237 & 959.452 & 959.716 & 959.772 \\
Estimated $r_{0}[\mathrm{~cm}]$ & 3.41 & 3.97 & 5.38 & 7.44 \\
Turbulence bias $\left.{ }^{\prime \prime}\right]$ & 0.54 & 0.41 & 0.16 & 0.06 \\
\hline Corrected radius $\left[{ }^{\prime \prime}\right]$ & 959.777 & 959.862 & 959.876 & 959.832 \\
Uncertainty $-1 \sigma{ }^{\left.{ }^{\prime \prime}\right]}$ & 0.194 & 0.185 & 0.129 & 0.105 \\
\hline
\end{tabular}

Notes. Results shown for each wavelength are obtained with quasiidentical exposure time. ${ }^{(a)}$ Refraction correction only.

\subsection{Discussion}

The SODISM II mean radius value without turbulence correction (959.24 arcsec) obtained at $535.7 \mathrm{~nm}$ (a) is slightly lower than the mean value obtained by the two historic instruments (Solar Astrolabe and DORAYSOL) installed at Calern observatory (959.51 arcsec). Unfortunately, Solar Astrolabe and DORAYSOL are no longer in operation and simultaneous measurements could not be made to date. The SODISM II mean radius value obtained at $535.7 \mathrm{~nm}(\mathrm{~b}, 8.9 \mathrm{~s})$ is slightly greater (50 mas) than $535.7 \mathrm{~nm}(\mathrm{a}, 1.3 \mathrm{~s})$, but the difference is within their uncertainties and they show the robustness of our instrumentation (almost equivalent results with two different interferential filters and with different exposure times). Thus, at $535.7 \mathrm{~nm}$, we find a solar radius of $959.78 \pm 0.19$ arcsec, which is slightly higher but still compatible with the canonical solar radius (Auwers 1891).

For SODISM II at $607.1 \mathrm{~nm}$, we found a solar radius of $959.86 \pm 0.18$ arcsec during the period 2011-2013. This result is also very similar to that obtained with the HMI instrument (959.90 \pm 0.06 arcsec) during the last Venus transit. These results obtained with different instruments and methods highlight a probable underestimation of the solar radius canonical value (959.63 arcsec) or a slight evolution since the eighteenth century. On the other hand, our results at all wavelengths lead us to suspect an overestimation of the solar radius obtained with the MDI instrument $(960.12 \pm 0.09$ arcsec). From radiative transfer simulations, we do not expect a variation of more than 20 mas between the measurements made at different wavelengths of the
Table 8. Difference ( $\triangle \mathrm{IPP})$ of the inflection-point position at $607.1 \mathrm{~nm}, 782.2 \mathrm{~nm}$, and $1025.0 \mathrm{~nm}$ from the IPP reference at $535.7 \mathrm{~nm}$ (a) for different solar models.

\begin{tabular}{lccc}
\hline \hline Wavelength $(\lambda)$ & $607.1 \mathrm{~nm}$ & $782.2 \mathrm{~nm}$ & $1025.0 \mathrm{~nm}$ \\
\hline$\Delta$ IPP VAL81 & 11.9 & 30.2 & - \\
$\Delta$ IPP FCH09 & 13.6 & 32.8 & - \\
$\Delta$ IPP SH09 & 9.4 & 21.2 & - \\
$\Delta$ IPP COSI & 10.0 & 28.0 & - \\
$\Delta$ IPP 3D & 13.5 & 25.0 & 21.0 \\
\hline SODISM II $\Delta$ IPP & 85.0 & 99.0 & 55.0 \\
\hline
\end{tabular}

Notes. SODISM II $\triangle$ IPP represents the values obtained by measurement. All values are in mas.

photospheric continuum. We have shown, however, that chromatic effects of astigmatism can contribute significantly to establish such a wavelength dependence in the measurements. From the ground, the bias introduced by optical turbulence is also dependent on the wavelength, and an inappropriate correction would also lead to different results.

At $1025.0 \mathrm{~nm}$, we initiated a new time-series and found a solar radius of 959.83 arcsec during the period 2011-2013. This wavelength is interesting because it is less sensitive to either optical turbulence and atmospheric absorption. Our detector performs less well in this spectral range, however, and we estimate that our best result in the present instrumental configuration is probably obtained at $782.2 \mathrm{~nm}$ (solar radius of $959.88 \pm 0.13$ arcsec).

The future work will be an even more detailed study of the instrumental and atmospheric turbulence effects and their uncertainty budget contribution. In addition, we have set up additional instrumentation to monitor various aspects of the instantaneous atmospheric conditions. A detailed analysis can also be conducted to verify the minor effects of aerosols on the solar radius measurements made by SODISM II. So far, we did not find any correlation between solar radius measurements and aerosol optical thickness or water vapor, which is less strong in winter. A seasonal effect still exists on the solar radius measurements after correction for refraction, but it is very likely related to the variation of the daily mean zenith distance, which enhances both the 

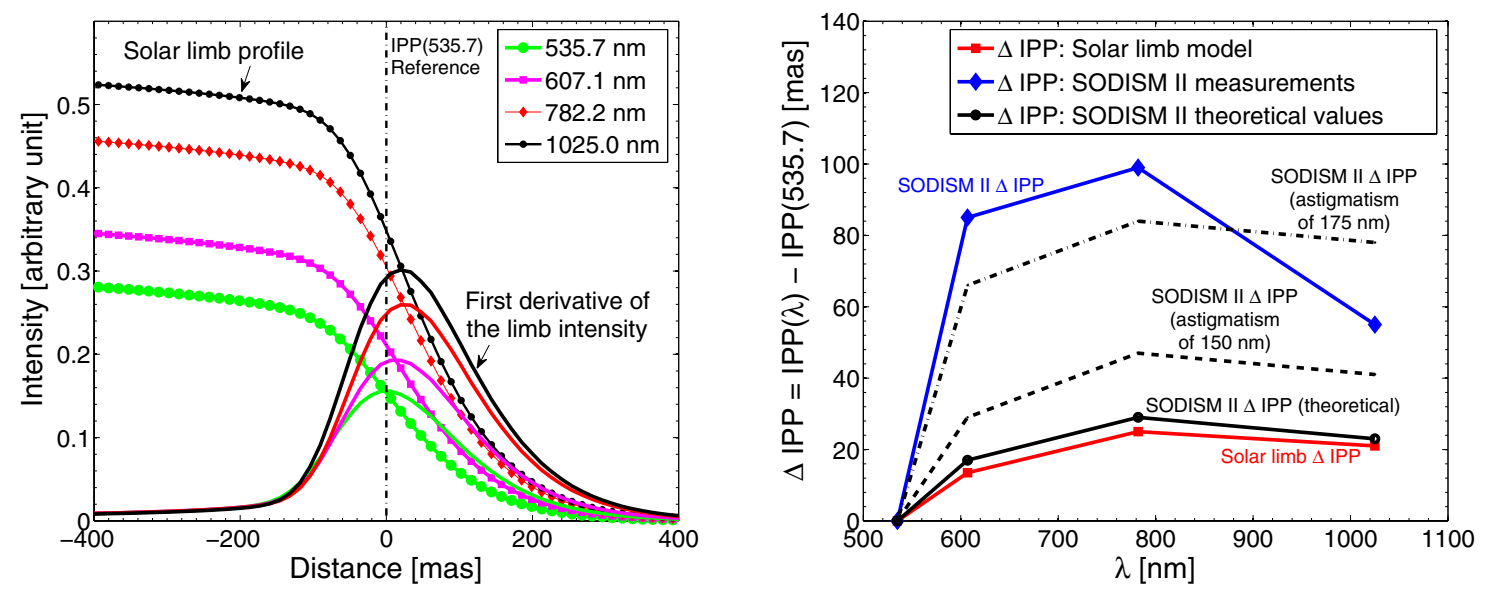

Fig. 15. Left: LDF at 535.7, 607.1, 782.2 and $1025.0 \mathrm{~nm}$, for theoretical solar models. First derivative of the LDF for each wavelength. Location of the IPP(535.7) reference position. Right: the red curve with circle symbol represents $\triangle$ IPP as a function of wavelength for 3D solar model. The blue curve with diamond symbols represents $\triangle$ IPP as a function of wavelength for SODISM II measurements. The black curves represents $\Delta$ IPP as a function of wavelength for SODISM II theoretical results (convolution between LDF and PSF for three different cases).

effects of atmospheric extinction and turbulence. The most important parameters are the refraction and the optical turbulence. Scattering of the solar light by aerosols is not dominant.

Solar cycle 24 is particularly low, and as mentioned above, we find no significant correlation between solar activity or TSI and the variation of the solar radius. This finding is the same as that performed by SDS or MDI (Sofia et al. 2013; Bush et al. 2010). During the rising phase of cycle 24 , however, we find much lower variations than those observed by SDS instrument over previous cycles. Only two and half years have been analyzed so far, but our first results show a remarkable stability at all wavelengths while the cycle was rising. This is consistent with the results obtained by MDI, which showed no significant variations of the solar radius in correlation or anticorrelation with activity over cycle 23 .

\section{Conclusion}

At Calern (France), a set of instruments was installed to carry out solar radius measurements with the capability of distinguishing the atmospheric effect that might affect them. These effects are turbulence and aerosols scattering. The photometer provides a quality index of pictures taken by SODISM II (aerosol optical thickness and water vapor). The pyranometer measures the solar irradiance at ground level and provides another quality index for SODISM II measurements. A camera also contributes to data selection. These instruments allow us to discard contaminated data. Because turbulence effects cannot be avoided, an instrument is dedicated to measure this. SODISM II provides a nearly continuous record of the solar radius at different wavelengths since May 2011. MISOLFA measures the spatio-temporal parameters of the local turbulence since 2010. Currently, given the noise affecting its measurements, only monthly averages are used. The average Fried parameter $r_{0}$ is $3.41 \mathrm{~cm}$ for the period of observations reported here. We showed that it is possible to quantify the effect of aerosols through an associated modulation transfer function (MTF) that can be multiplied to the optical turbulence MTF to estimate the overall atmospheric MTF (e.g., Dror \& Kopeika 1995). Therefore aerosols potentially have an impact on image spread, but it is expected to be small when observing the Sun from the heated ground where most of the optical turbulence is generated. Our first results presented here confirm that to first order, the effect of aerosols is rather only extinction and that optical turbulence is the only significant effect that contributes to the image spread. A detailed analysis of the PAPS records will help us in the future to determine the potential minor effects caused by aerosols. In this work, data selection was made mainly with the PAPS, PPS, and CPS. We are still working to improve the MISOLFA signal-to-noise ratio and pipeline, however, to derive estimates of $r_{0}$ every minute. We have shown (Fig. 7 of Ikhlef et al. 2012) that at given moments the $r_{0}$ value can be as high as $8 \mathrm{~cm}$, which is significantly higher than the monthly average value. From Fig. 5, we see that the bias introduced by turbulence is low and remains roughly constant for $r_{0}$ above $7 \mathrm{~cm}$. Selecting these particular images would therefore effectively lead to a series with a lower bias introduced by turbulence and lower uncertainties associated with the estimated bias corrections. Main corrections to the observed radius of the Sun are (i) atmospheric refraction (at most $\sim 1.0$ arc$\mathrm{sec}$ ); and (ii) seeing, which generates a bias lower than $\sim 0.5$ arcsec, which is wavelength dependent. The SODISM II solar radius at $535.7 \mathrm{~nm}$, after making all necessary corrections, is close to 959.78 arcsec, while the estimated uncertainties of the measurements are typically smaller than 0.2 arcsec. For other wavelengths of the SODISM II instrument, the solar radii are close to 959.9 arcsec. The solar radius observed with SODISM II (during the period 2011-2013) results from variations smaller than 50 mas and it is out of phase with solar activity. A continuation of PICARD ground-based segment will allow us to confirm our observations about the variations of the solar radius.

Acknowledgements. This project is a collaboration between LATMOS, OCA, and CRAAG and is supported by CNES and CNRS. We thank CNES (Centre National d'Études Spatiales), CNRS (Centre National de la Recherche Scientifique), Laboratoire d'Optique Atmosphérique (University of Lille 1), and LESIA (Laboratoire d'Études Spatiales et d'Instrumentation en Astrophysique) for their support as well as all the participants who have devoted their expertise to this project. Numerous individuals have been involved in this project. They are all gratefully acknowledged for their respective contributions, although they cannot be listed here because many of them joined the development for only few months. The following institutes are acknowledged for providing the data: Solar Influences Data Center (Belgium), the "Physikalisch-Meteorologisches Observatorium Davos" (PMOD, Switzerland) and the Hansen Experimental Physics Laboratory (HEPL, Stanford University, United Sates). We especially 
want to thank Rock Bush (HEPL team, Stanford University) who provided data from the MDI instrument. We also thank Alexander Shapiro and Werner Schmutz (PMOD) who provided COSI solar models at wavelengths associated with our instrument. We would like to thank Farrokh Vakili (Head of Côte d'Azur Observatory) for his support and provision of Calern site means. Finally, we thank the referee and the Editor for the constructive remarks and suggestions. This work is dedicated to the memory of Francis Laclare, who inspired a generation of astrophysicist to consider the measurement and interpretation of the solar diameter.

\section{References}

Adassuriya, J., Gunasekera, S., \& Samarasinha, N. 2011, Sun and Geosphere, 6, 17

Assus, P., Borgnino, J., Martin, F., et al. 2002, in Astronomical Site Evaluation in the Visible and Radio Range, eds. J. Vernin, Z. Benkhaldoun, \& C. MuñozTuñón, ASP Conf. Ser., 266, 134

Auwers, A. 1891, Astron. Nachr., 128, 361

Badache-Damiani, C., Rozelot, J. P., Coughlin, K., \& Kilifarska, N. 2007, MNRAS, 380, 609

Ball, W. T., Unruh, Y. C., Krivova, N. A., et al. 2012, A\&A, 541, A27

BenMoussa, A., Gissot, S., Schühle, U., et al. 2013, Sol. Phys., 288, 389

Braun, H., Christl, M., Rahmstorf, S., et al. 2005, Nature, 438, 208

Brown, T. M. 1982, A\&A, 116, 260

Brown, T. M., \& Christensen-Dalsgaard, J. 1998, ApJ, 500, L195

Bush, R. I., Emilio, M., \& Kuhn, J. R. 2010, ApJ, 716, 1381

Corbard, T., Irbah, A., Assus, P., et al. 2010, Astron. Nachr., 331, P58

Coulter, R. L., Kuhn, J. R., \& Lin, H. 1996, in BAAS 28, 912

Delache, P., \& Kroll, R. J. 1994, Booktitle: The Solar Engine and its Influence on Terrestrial Atmosphere and Climate, ed. Nesme-Ribes, E. (Berlin, Heidelberg: Springer Verlag), 193

Dror, I., \& Kopeika, N. S. 1995, J. Opt. Soc. Am. A, 12, 970

Duchon, C. E., \& O’Malley, M. S. 1999, J. Appl. Meteorol., 38, 132

Eddy, J. A., \& Boornazian, A. A. 1979, in BAAS, 11, 437

Emilio, M., Kuhn, J. R., Bush, R. I., \& Scholl, I. F. 2012, ApJ, 750, 135

Fontenla, J. M., Curdt, W., Haberreiter, M., Harder, J., \& Tian, H. 2009, ApJ, 707,482

Foukal, P., Fröhlich, C., Spruit, H., \& Wigley, T. M. L. 2006, Nature, 443, 161

Gilliland, R. L. 1981, ApJ, 248, 1144

Haberreiter, M., Schmutz, W., \& Hubeny, I. 2008a, A\&A, 492, 833

Haberreiter, M., Schmutz, W., \& Kosovichev, A. G. 2008b, ApJ, 675, L53

Hauchecorne, A., Meftah, M., Irbah, A., et al. 2014, ApJ, 783, 127

Hestroffer, D., \& Magnan, C. 1998, A\&A, 333, 338
Hochedez, J.-F., Timmermans, C., Hauchecorne, A., \& Meftah, M. 2014, A\&A, 561, A 17

Holben, B. N., Tanré, D., Smirnov, A., et al. 2001, J. Geophys. Res., 106, 12067

Ikhlef, R., Corbard, T., Irbah, A., et al. 2012, in SPIE Conf. Ser. 8444

Irbah, A., Chibani, M., Lakhal, L., et al. 2001, in SF2A-2001: Semaine de l'Astrophysique Française, eds. F. Combes, D. Barret, \& F. Thévenin, 59

Irbah, A., Corbard, T., Assus, P., et al. 2010, in SPIE Conf. Ser., 7735

Kuhn, J. R., Lin, H., \& Loranz, D. 1991, PASP, 103, 1097

Kuhn, J. R., Bush, R. I., Emilio, M., \& Scherrer, P. H. 2004, ApJ, 613, 1241

Kuhn, J. R., Bush, R., Emilio, M., \& Scholl, I. F. 2012, Science, 337, 1638

Laclare, F., Delmas, C., Coin, J. P., \& Irbah, A. 1996, Sol. Phys., 166, 211

Laclare, F., Delmas, C., Sinceac, V., \& Chollet, F. 1999, Académie des Sciences Paris Comptes Rendus Série B Sciences Physiques, 327, 645

Lakhal, L., Irbah, A., Bouzaria, M., et al. 1999, A\&AS, 138, 155

Meftah, M., Irbah, A., Corbard, T., et al. 2012, in SPIE Conf. Ser., 8446

Meftah, M., Hauchecorne, A., Crepel, M., et al. 2014a, Sol. Phys., 289, 1

Meftah, M., Hochedez, J.-F., Irbah, A., et al. 2014b, Sol. Phys., 289, 1043

Morand, F., Delmas, C., Corbard, T., et al. 2010, C. R. Phys., 11, 660

Parkinson, J. H., Morrison, L. V., \& Stephenson, F. R. 1980, Nature, 288, 548

Piau, L., Stein, R. F., Melo, S., et al. 2011, in SF2A-2011: Proc. Annual meeting of the French Society of Astronomy and Astrophysics, eds. G. Alecian, K. Belkacem, R. Samadi, \& D. Valls-Gabaud, 407

Ribes, E., Beardsley, B., Brown, T. M., et al. 1991, Booktitle: The Sun in Time, eds. C. P. Sonett, \& M. S. Giampapa, \& M. S. Matthews (Tucson: Univerisity of Arizona Press), 59

Rozelot, J. P. 2001a, J. Atmos. Sol.-Terr. Phys., 63, 375

Rozelot, J. P. 2001b, J. Atmos. Sol.-Terr. Phys., 63, 2005

Rozelot, J. P., \& Damiani, C. 2012, EPJH, 37, 709

Scherrer, P. H., Schou, J., Bush, R. I., et al. 2012, Sol. Phys., 275, 207

Schou, J., Scherrer, P. H., Bush, R. I., et al. 2012, Sol. Phys., 275, 229

Schröder, W. 2001, J. Atmos. Sol.-Terr. Phys., 63, 2003

Shapiro, A. I., Schmutz, W., Schoell, M., Haberreiter, M., \& Rozanov, E. 2010, A\&A, 517, A48

Short, C. I., \& Hauschildt, P. H. 2009, ApJ, 691, 1634

Sofia, S., Chiu, H.-Y., Maier, E., et al. 1984, Appl. Opt., 23, 1235

Sofia, S., Girard, T. M., Sofia, U. J., et al. 2013, MNRAS, 436, 2151

Spruit, H. C. 1991, in The Sun in Time, eds. C. P. Sonett, M. S. Giampapa, \& M. S. Matthews (Tucson: University of Arizona Press), 118

Thuillier, G., Dewitte, S., \& Schmutz, W. 2006, Adv. Space Res., 38, 1792

Thuillier, G., Claudel, J., Djafer, D., et al. 2011, Sol. Phys., 268, 125

Toulmonde, M. 1997, A\&A, 325, 1174

Vernazza, J. E., Avrett, E. H., \& Loeser, R. 1981, ApJS, 45, 635

Wittmann, A. 1977, A\&A, 61, 225 